\title{
Use of Endoluminal Ultrasound to Evaluate Gastrointestinal Motility
}

\author{
Larry Miller Qing Dai Annapurna Korimilli Barry Levitt Zeeshan Ramzan \\ James Brasseur \\ Temple University Hospital, Gastroenterology Section, Philadelphia, Pa., USA
}

\section{Key Words}

Endoluminal ultrasound $\cdot$ Motility $\cdot$ Esophageal motility disorders · High-frequency ultrasound • Three-dimensional ultrasound

\begin{abstract}
The use of high-frequency ultrasound transducers in the gastrointestinal tract (GI) has already yielded remarkable findings concerning the anatomy, physiology and pathophysiology of the GI tract and of various motility disorders. These transducers have made completely invisible portions of the GI tract (the longitudinal smooth muscle, muscles of the upper esophageal sphincter, components of the gastroesophageal junction high-pressure zone, and the muscle of the anal sphincter complex) accessible to investigation. Use of simultaneous ultrasound and manometry has allowed the exploration of the normal physiology of peristaltic contraction. The components of the high-pressure zone of the distal and proximal esophagus have been isolated and the movement of these components has been studied individually and as a group. Various esophageal motility disorders have been investigated including achalasia, scleroderma, Barrett's esophagus and diffuse esophageal spasm. The possible etiology of the symptoms of esophageal chest pain and heartburn (sustained esophageal contractions of the longitudinal smooth muscle), have been studied. The pos-
\end{abstract}

sible underlying pathophysiology of GERD (the missing gastric clasp and sling fiber pressure profile) has been explored. Three-dimensional high-frequency ultrasound imaging has allowed the peristaltic contraction sequence to be viewed in a completely new and unique manner. The biomechanics of both esophageal contraction and the gastroesophageal junction high-pressure zone have been investigated and the mechanical advantage of esophageal shorting has been studied. The mechanism of action of standard surgical and newer endoscopic therapies for GERD has been defined. Future applications of this technology are limited only by our imagination.

Copyright $(2006$ S. Karger AG, Basel

\section{Introduction}

Over the past several years, high-frequency (12.5$20 \mathrm{MHz}$ ) catheter-based ultrasound transducers (4.8$9 \mathrm{Fr}$ ), originally designed for intravascular ultrasound applications, have become available to investigators studying the gastrointestinal tract and gastrointestinal motility.

Miller and associates [1-3] were the first to study esophageal motility using ultrasound. The catheter-based ultrasound transducers used for these studies differ from the routinely used endoscopic ultrasound transducers in

\section{KARGER}

Fax +41613061234

E-Mail karger@karger.ch

www.karger.com
(C) 2006 S. Karger AG, Basel

$0257-2753 / 06 / 0244-0319 \$ 23.50 / 0$

Accessible online at:

www.karger.com/ddi
Larry Miller, MD

Temple University Hospital, Gastroenterology Section

8th Floor, Parkinson Pavillion

Philadelphia, PA 19043 (USA)

Tel. +1 215707 6922, Fax +1 215707 2684, E-Mail larry.miller@temple.edu 


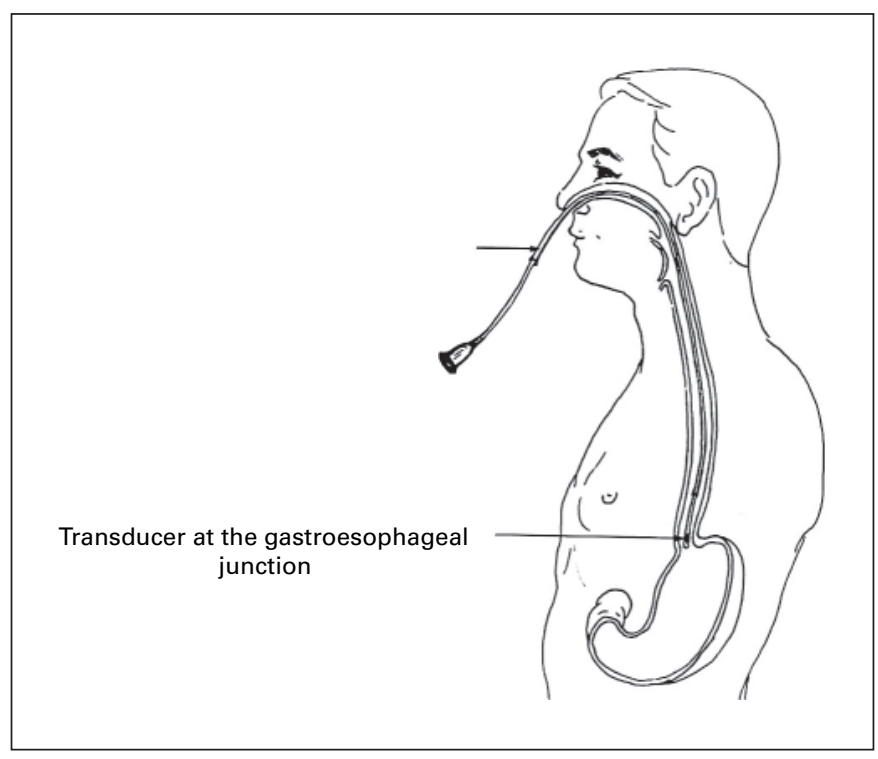

Fig. 1. The ultrasound transducer being passed transnasally into the distal esophagus.

a number of ways. First, the size of the ultrasound catheter is much smaller than the dedicated endoscopic ultrasound transducers. Second, the frequency used (20 MHz) is much higher than the frequency used in standard echoendoscopes $(5-12 \mathrm{MHz})$. The higher frequency increases the axial resolution of the image so that mural gastrointestinal structures, especially the muscle layers, can be seen with much greater resolution. Third, these transducers are not typically surrounded by a water-filled balloon as are the transducers embedded within dedicated endosonoscopes. There are certain advantages and disadvantages to this. Because of the smaller size and the lack of compression, there is much less distortion of small mural structures and of the muscle layers within the wall of the gastrointestinal tract. The disadvantage is that there may be loss of acoustic coupling and therefore ultrasound imaging, in organs filled with air, such as the stomach, the small intestine and the esophagus during certain phases of swallowing.

\section{Ultrasound Technology}

In general, the high-frequency ultrasound probe catheters are available in different diameters $(1-3 \mathrm{~mm})$ and various lengths $(95-150 \mathrm{~cm})$. The ultrasound frequency of the transducers ranges from 9 to $40 \mathrm{MHz}$. As the transducer frequency increases, the depth of penetration de- creases but the image resolution increases. The depth of penetration of a $20-\mathrm{MHz}$ frequency is approximately $2 \mathrm{~cm}$. Two types of transducers that produce cross-sectional images are currently in use: (1) mechanical transducers in which the transducer, located on the tip of the shaft, rotates $360^{\circ}$ at a speed of $5-40 \mathrm{~Hz}$ and (2) a synthetic aperture type of transducer that uses piezoelectric elements that are circumferentially placed around a 1.2-mm catheter (fig. 1). Seven to nine different layers of the esophageal wall can be discerned in the ultrasound images with a $20-\mathrm{MHz}$ transducer. The anatomy of these layers, especially the two layers of the muscularis propria, change in a dynamic fashion during esophageal contraction. Because ultrasound images are recorded in real time, high-frequency endoluminal ultrasound provides a unique way to study the dynamics of motility in the gastrointestinal tract.

\section{Simultaneous Endoluminal Ultrasound and Manometry}

Because the diameter of the high-frequency ultrasound catheter is relatively small the ultrasound transducer may be combined with a manometry catheter to study the relationship between anatomic structures and physiologic and pathophysiologic events. Simultaneous ultrasound and manometry was developed by Miller et al. [4] to study the physiology (pressure) and anatomy (muscle thickness cross-sectional area) of peristaltic contractions simultaneously in an effort to better understand the mechanics involved in swallowing (fig. 2). This technology has subsequently been used to evaluate the gastroesophageal junction high-pressure zone [5], the anal sphincter complex [6] and various esophageal motility disorders.

\section{Three-Dimensional Ultrasound Technology}

The use of 3-dimensional technology in ultrasonography was made possible by recent advances in computer technology [7]. Endoluminal ultrasound in particular lends itself to 3-dimensional reconstruction. Three-dimensional ultrasound images are created by stacking twodimensional slices. Most 3-dimensional acquisition techniques share a common feature; during a scanning procedure, a data set of 2-dimensional ultrasound images, along with their relative spatial locations and orientations, are saved in a computer. Knowledge of the spatial location and orientation of the scanned planes allows the 

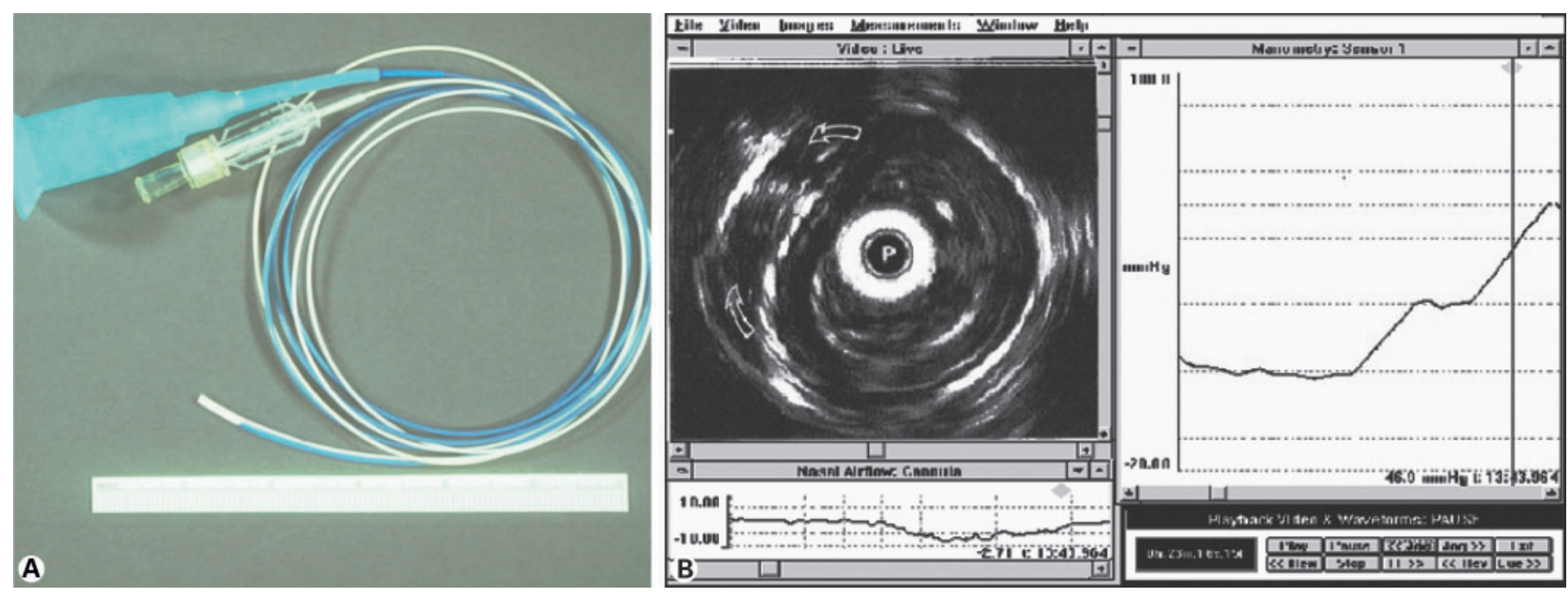

Fig. 2. A Simultaneous ultrasound and manometry catheter. The blue catheter is the ultrasound catheter, which contains the ultrasound transducer. The white catheter is the manometry catheter. B Ultrasound image and the corresponding manometric pressure $(46.0 \mathrm{~mm} \mathrm{Hg})$ and respiratory cycle (nasal airflow cannula). Note that the ultrasound image on the left is occurring at the same time, indicated by the vertical line, as the manometry tracing on the right. This allows synchronization of the ultrasound image with the pressure generated by the muscles in that image.

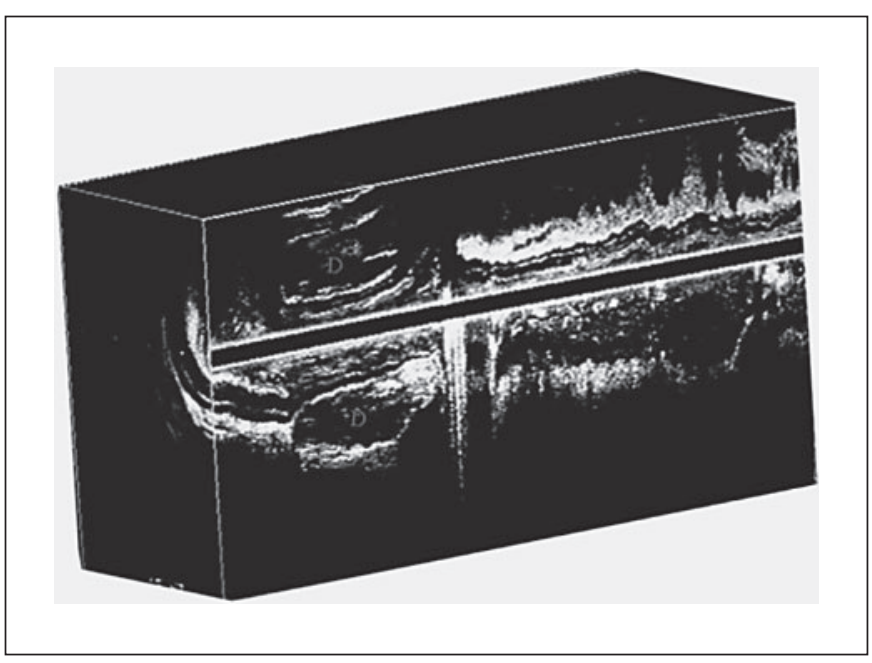

Fig. 3. Three-dimensional construct of the distal esophagus. The crural diaphragm is labeled D.

visualization program to reconstruct the original geometry of the scanned object (fig. 3).

Prior 3-dimensional acquisition techniques were all based on the common principal of moving the ultrasound transducer and then reconstructing the 3-dimensional image by spatial orientation of the 2-dimensional images.
Recently, Dai et al. [8] developed a new 3-dimensional acquisition technique used to study esophageal motility in which the transducer was kept stationary and the esophagus moved around the transducer during swallowing. Thus a new technique to investigate peristaltic contraction was created.

\section{Evaluation of the Normal Esophagus}

\section{Introduction}

The initial gastrointestinal studies performed using 20-MHz catheter-based ultrasound transducers were animal studies using sheep esophagi [1]. Esophageal specimens were studied in vitro. The fresh specimens were placed in a water bath, and the transducer-containing catheter was inserted in the lumen of the esophagus. After ultrasound imaging, histologic cross sections were prepared that corresponded to the ultrasound imaging planes. The recorded ultrasound images demonstrated seven layers of the esophageal wall. Correlation of the histologic findings with these ultrasound images showed that the innermost (hyperechoic) layer represented the squamous epithelium and lamina propria; the second (hypoechoic) layer the muscularis mucosa and deep mucosa; the third (hyperechoic) layer the submucosa; the fourth (hypoecho- 


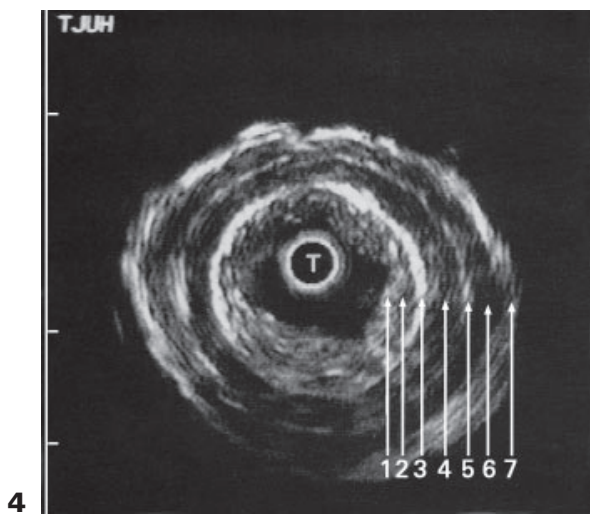

Fig. 4. Cross-sectional ultrasound image from the post-mortem fluid-filled esophagus in a patient that died of non-gastrointestinal related disease. Correlation of the histologic findings with these ultrasound images showed that the innermost (hyperechoic) layer (1) represented the squamous epithelium and lamina propria, the second (hypoechoic) layer (2) the muscularis mucosa and deep mucosa, the third (hyperechoic) layer (3) the submucosa, the fourth (hypoechoic) layer (4) the circular muscle, the fifth (hyperechoic) layer (5) the intramuscular connective tissue, the sixth (hypoecho-

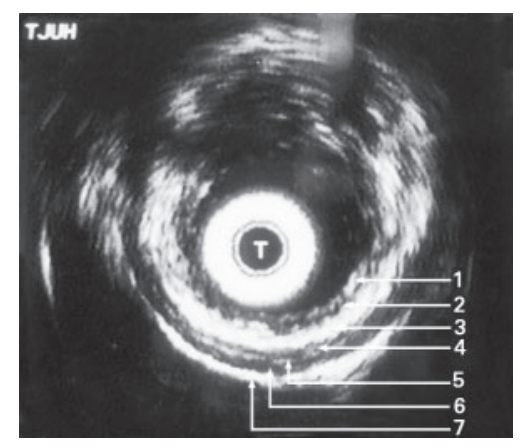

Fig. 6. The esophagus at a level $10 \mathrm{~cm}$ above the LES during bolus distension of the esophagus with water. Note that there are now seven wall layers seen. The innermost (hyperechoic) layer represented the squamous epithelium and lamina propria, the second (hypoechoic) layer the muscularis mucosa and deep mucosa, the third (hyperechoic) layer the submucosa, the fourth (hypoechoic) layer the circular muscle, the fifth (hyperechoic) layer the intramuscular connective tissue, the sixth (hypoechoic) layer the longitudinal muscle, and the seventh (hyperechoic) layer the adventitia.

ic) layer the circular muscle; the fifth (hyperechoic) layer the intramuscular connective tissue; the sixth (hypoechoic) layer the longitudinal muscle, and the seventh (hyperechoic) layer the adventitia.

The human esophagus was first evaluated using in vitro autopsy specimens [2]. These specimens were infused with various volumes of water and placed in a water bath. ic) layer (6) the longitudinal muscle, and the seventh (hyperechoic) layer (7) the adventitia.

Fig. 5. A 20-MHz endoluminal ultrasound scan showing the esophagus at the level of the LES (A) and approximately $10 \mathrm{~cm}$ above the LES (B). The configuration of the esophagus at this level is due to compression from extra esophageal structures. $\mathrm{T}=$ Transducer; 1 = mucosa; 2 = submucosa; 3 = circular smooth muscle; 4 = intramuscular connective tissue; 5 = longitudinal smooth muscle; $6=$ adventitia.

The catheter containing the ultrasound transducer was introduced into the esophagus and an image of the entire esophagus was obtained. The esophagus was then sectioned for histologic evaluation. The histologic observations on cross section were compared with the cross-sectional endoluminal ultrasound image at the corresponding levels. Analysis of images obtained revealed seven reproducible and distinct alternating hyperechoic and hypoechoic layers in the esophageal wall which were comparable with the animal studies above (fig. 4).

After demonstrating its ability to provide high-resolution imaging of the esophageal wall in both an in vitro animal esophageal model and in human esophageal autopsy specimens, Miller et al. [1] performed in vivo studies in normal human volunteers. All of the layers seen in the autopsy specimens were seen in the in vivo human esophagus. When the esophagus was at rest, six layers were seen, similar to the non-fluid-filled autopsy esophageal specimens (fig. 5). During the swallowing of a 5- to 10 -ml water bolus, seven layers were seen, similar to the fluid-filled autopsy esophageal specimens (fig. 6). A number of other findings were also consistently seen on imaging of the normal volunteers' esophagi. First, in the area of the lower esophageal sphincter (LES), the muscle layers were found to be significantly thicker than in the body of the esophagus. Second, the area of the LES was consistently round, and the diaphragm was consistently seen in that area as a hypoechoic band just outside the wall of the 

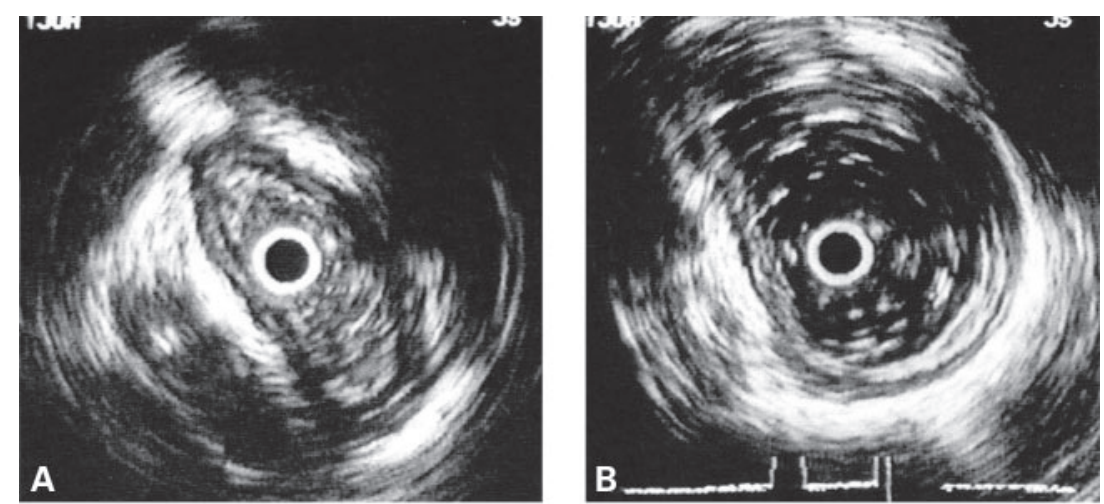

Fig. 7. A Sonogram obtained during the resting phase of the peristaltic sequence, when the muscularis propria is at rest. B Sonogram obtained during the passive distention phase of the peristaltic sequence. Water can be seen within the lumen of the esophagus, and the muscle width is decreased. C Sonogram obtained during the contraction sequence of peristalsis, in which there is a significant increase in muscle thickness. D Sonogram obtained during the relaxation phase of the peristaltic sequence, in which the muscle thickness once again returns to the thickness of the resting phase.
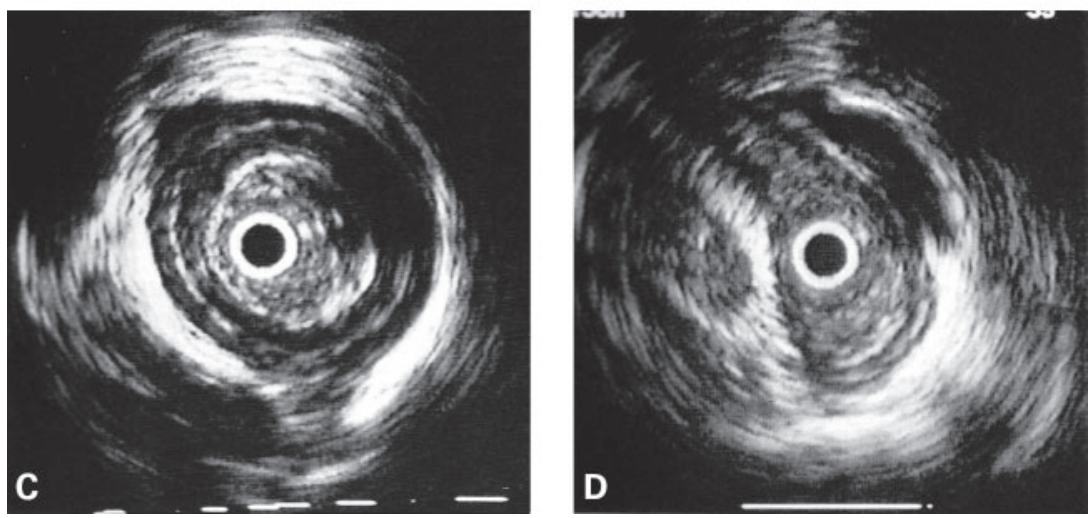

esophagus. However, in its mid- and upper portions, the esophagus was slit-like and asymmetric. This configuration appeared to be secondary to compression from various periesophageal structures. Third, the submucosa was consistently brighter, or more hyperechoic in the area just below the upper esophageal sphincter than in the body or the region of the LES. Fourth, the upper esophageal sphincter was consistently demonstrated in every subject. It was also found that the circular smooth muscle was significantly thicker then the longitudinal smooth muscle, both at the level of the LES and $5-10 \mathrm{~cm}$ above the LES.

\section{Esophageal Body and Peristaltic Contraction}

Sequential ultrasound imaging was obtained in the body of the esophagus during the swallowing of 5- to 10$\mathrm{ml}$ boluses of water in normal volunteers [1]. At rest the esophagus appeared slit-like and asymmetric. When the lumen was distended, there was an associated decrease in the esophageal wall thickness. This was most marked in the two muscular layers. Following the distension, the two smooth muscle layers contracted and the lumen narrowed, resulting in increased wall thickness. During this phase the esophagus became round and symmetric and the circular smooth muscle layer increased in echogenicity. Subsequently the esophagus returned to the resting state.

Additional studies were conducted to correlate the changes in muscle width and lumen circumference to changes in intraluminal pressure and to define various sonographic phases of the swallowing sequence based on manometrically defined pressure changes in the esophageal lumen. Miller et al. [4] devised a probe that consisted of a $20-\mathrm{MHz}$ high-frequency ultrasonography transducer attached to a manometric catheter so that ultrasound images and manometry pressure could be obtained simultaneously (fig. 2). The LES was located manometrically and the catheter was placed at $10 \mathrm{~cm}$ proximal to the LES. Each of the subjects was evaluated during 10-20 swallows. The resulting images were then analyzed.

Ultrasonography revealed four phases of esophageal peristalsis in the esophageal body at a site $10 \mathrm{~cm}$ above the LES. Phase 1 was the resting phase, which occurred before the bolus reached the ultrasound transducer (fig. $7,8)$. During phase 1 , the circular and longitudinal smooth muscle appeared as thin circumferential hypoechoic bands separated by a thin hyperechoic intramuscular connective tissue. The contour of the esophageal wall was 
influenced by compression caused by surrounding structures. In this phase, the manometric pressure did not vary from the baseline pressure.

Phase 2 was the phase during which there was passive stretching and luminal opening of the esophagus by the bolus. As the bolus progressed past the transducer, the lumen opened progressively, and the muscularis propria

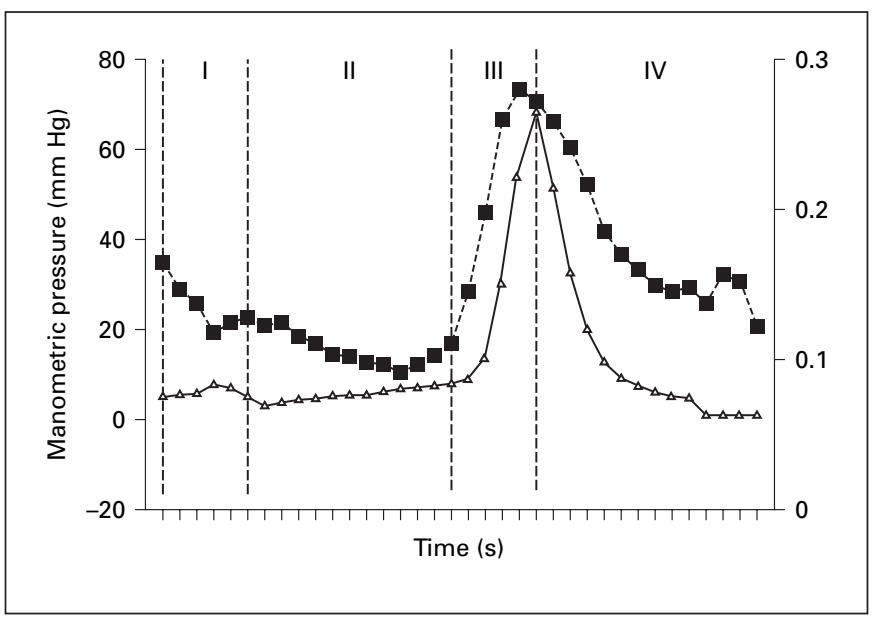

Fig. 8. Manometric pressure $(\triangle)$ vs. muscle thickness $(\boldsymbol{\square})$. The peak pressure and peak muscle thickness occur at approximately the same time. layers progressively decreased in width. The width of the circular smooth muscle, longitudinal smooth muscle, and total muscle thickness decreased during the passive stretching phase to their thinnest muscle width. The pressure recorded on the manometry tracing remained at the baseline resting pressure.

Phase 3 was the contraction phase that began at the start of the positive deflection on the manometry tracing. During this phase, the lumen circumference rapidly decreased in diameter to zero, and the entire esophagus took on a rounded appearance as the muscle contracted. Both the circular smooth muscle and longitudinal smooth muscle became progressively thicker, and reached their mean maximum widths during this phase. Maximal mean muscle thickness occurred when the manometric tracing reached a maximum peak pressure. This phase of the contraction sequence is analogous to the previously described tail of the peristaltic stripping wave that can be observed on barium esophagography to have a close relationship with the onset of the upstroke of the pressure wave.

Phase 4 was the relaxation phase that began with a decline in the manometric pressure from its peak pressure. During this last phase, the esophagus lost its rounded appearance and took on an irregular shape similar to phase 1 . During this phase, the lumen circumference remained zero. The muscle relaxed with a decrease in mus-
Fig. 9. Three-dimensional reconstruction of a peristaltic contraction sequence. The transducer was held steady while the esophagus moved. The $\mathrm{X}$-axis represents time. All of the images used in this 3-dimensional reconstruction are taken at the same spatial location within the esophagus. The manometric phases of the peristaltic contraction sequence can be related to phases on the 3-dimensional reconstruction. Twodimensional slices are shown on top.

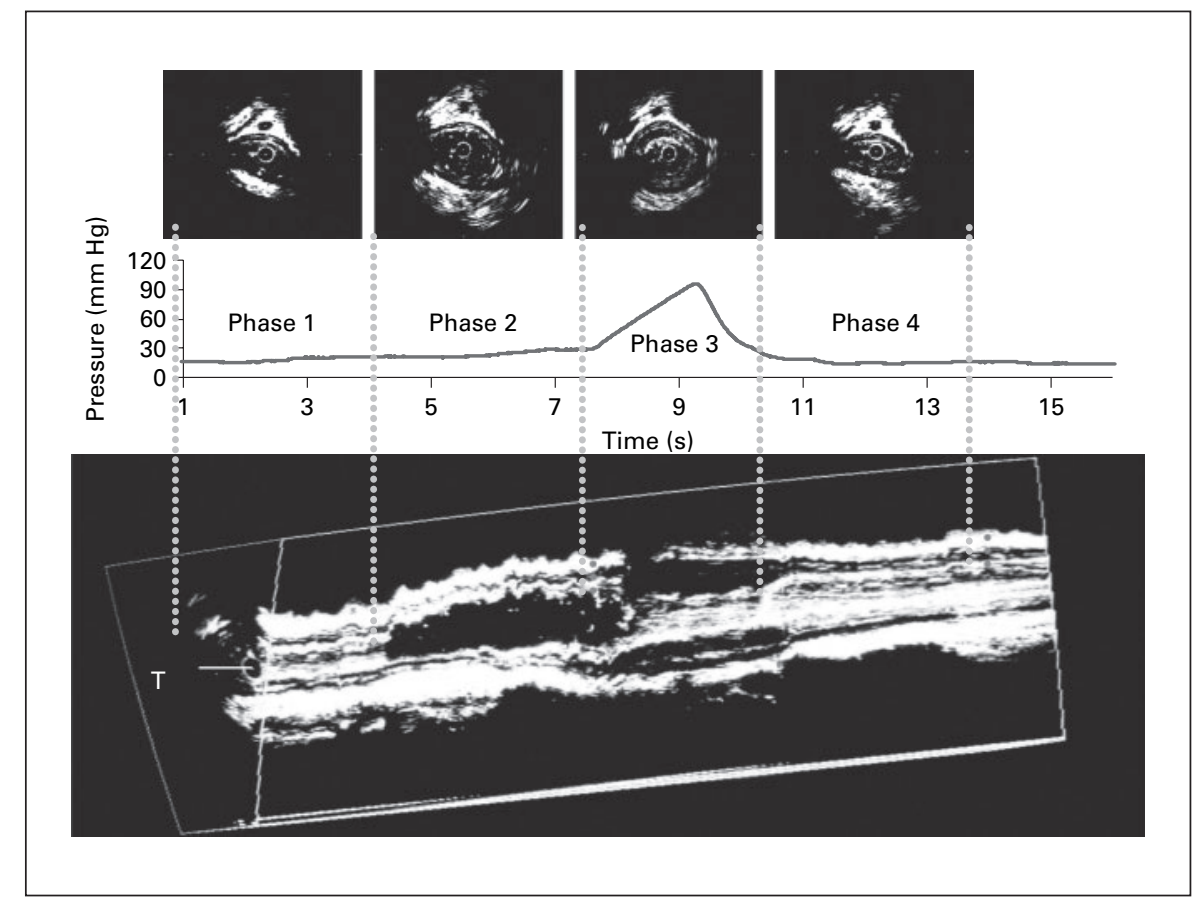

Miller/Dai/Korimilli/Levitt/Ramzan/ Brasseur 
cle thickness back to baseline resting thickness, as well as a return of intraesophageal pressures back to baseline.

Dai et al. [8] developed a technique in which 3-dimensional (space-time) reconstructions were acquired during peristaltic contraction. Two-dimensional ultrasound images were reconstructed in 3-dimensional space-time plots with time as the $\mathrm{X}$-axis variable. This allowed for the dynamics of the peristaltic contraction to be viewed in a unique manner so that the physiology of the contraction could be evaluated simply and intuitively (fig. 9). By viewing these images, the lumen circumference, muscle thickness, changes in geometry, and changes in pressure could be evaluated simultaneously and related to each other, and the various phases of the peristaltic contraction sequence could be studied. The reconstructed 3-dimensional time-resolved images of the peristaltic contraction sequence demonstrated various layers of the esophageal wall. By cutting through the image longitudinally, the various layers of the esophageal wall could be followed and studied during the entire peristaltic contraction sequence demonstrating the same four phases previously described [9].

\section{Evaluation of Longitudinal Smooth Muscle}

Nicosia et al. [10] analyzed local longitudinal shortening of the esophageal body by combining concurrent ultrasonography and manometry with basic principles of mechanics. They applied the law of mass conservation to quantify local axial shortening of the esophageal wall from ultrasonically measured cross-sectional area concurrently with measured intraluminal pressure, from which correlations between local contraction of longitudinal and circular muscle were inferred (fig. 10). Two clear phases of local longitudinal shortening were observed during bolus transport. During luminal filling by bolus fluid, the muscle layer distended and the muscle thickness decreased in the absence of circular or longitudinal muscle contraction. This was followed by local contraction, first in longitudinal muscle, then in circular muscle (fig. 11). Maximal longitudinal shortening occurred nearly coincidently with peak intraluminal pressure. Longitudinal muscle contraction began before and ended after circular muscle contraction. Larger longitudinal shortening was correlated with higher-pressure amplitude, suggesting that circumferential contractile forces were enhanced by longitudinal muscle shortening. These results suggested that the coordination of the two waves of muscle contraction (circular smooth muscle and longitudinal smooth muscle) may be a physiological response to the mechanical influence of longitudinal shortening,

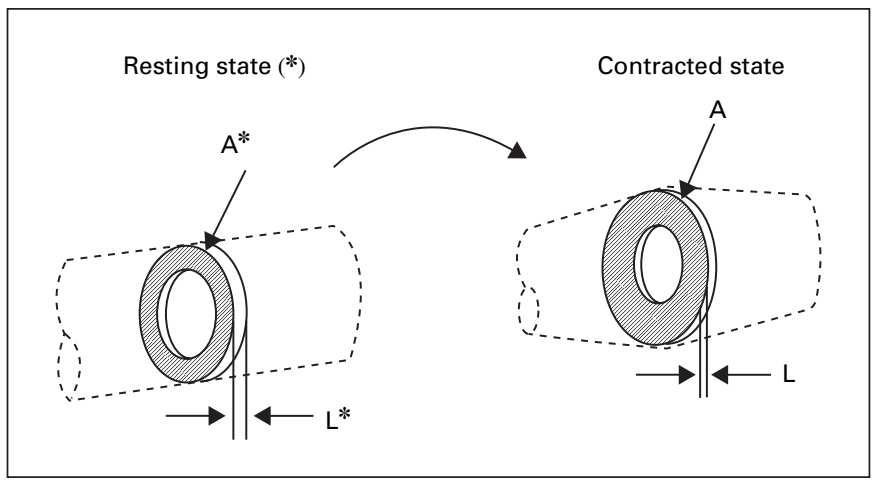

Fig. 10. Relationship between local muscle shortening and the changes in the cross-sectional area of esophageal muscle, where $A^{*}$ and $\mathrm{A}$ are the cross-sectional areas at the resting phase and at the contracting phase, respectively, $\mathrm{L}^{*}$ and $\mathrm{L}$ are the lengths of esophageal muscle at rest and at the contraction, respectively. The law of the mass conservation implies $\mathrm{L}^{*} \mathrm{~L}^{*}=\mathrm{A}^{*} / \mathrm{A}$, where $\mathrm{A}^{*} / \mathrm{A}$ can be measured from digitized ultrasound images to quantify $\left.\mathrm{L} / \mathrm{L}^{*}\right)$. From Nicosia et al. [10].

which increases contractile force while reducing average muscle fiber tension by increasing circular muscle fiber density locally near the bolus tail [11].

Gastroesophageal Junction High-Pressure Zone of the Distal Esophagus (LES, Crural Diaphragm, Gastric

Sling and Clasp Fibers)

McCray et al. [5] were the first to use simultaneous ultrasound and manometry to evaluate the high-pressure zone of the distal esophagus and differentiate the pressure profile generated by the intrinsic esophageal sphincter from the pressure profile generated by the crural diaphragm. In the initial studies, normal volunteers were evaluated using a simultaneous ultrasound/manometry catheter. The catheter was positioned in the proximal stomach and then pulled through the high-pressure zone at a constant velocity during maximal end inspiration. Manometric pressures and ultrasound images were recorded. The cross-sectional surface area on sonographic images of the intrinsic esophageal sphincter (LES) and crural diaphragm, and the manometric pressures of the high-pressure zone were plotted against time. The highpressure zone in the distal esophagus was characterized sonographically as the crural diaphragm distally, as an overlap of the crural diaphragm and LES more proximally and as the intrinsic LES alone in the most proximal portion of the gastroesophageal junction high-pressure zone (fig. 12). In all volunteers, peak pressure corresponded to an overlap of the crural diaphragm and the LES. 


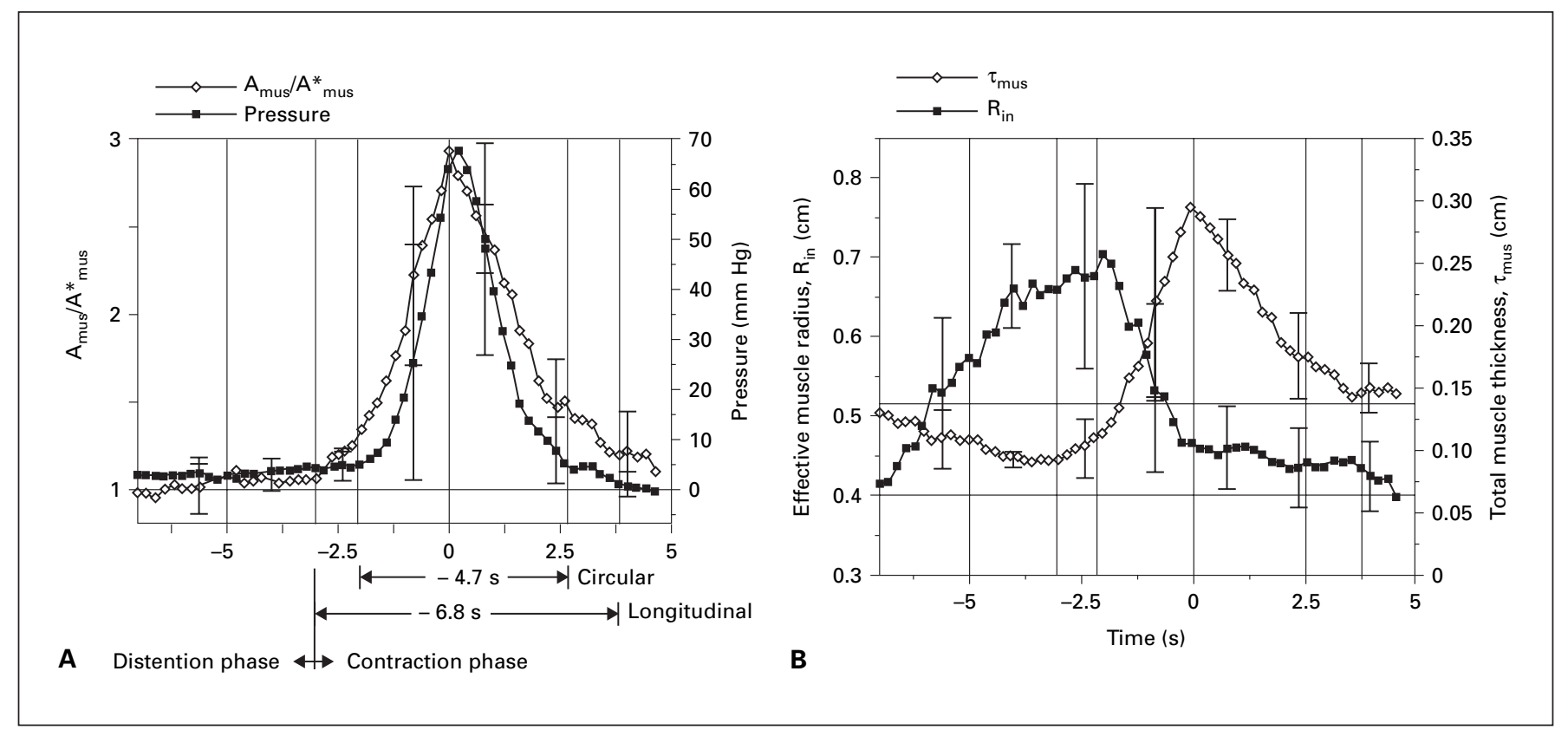

Fig. 11. Temporal variation in total muscle area $A_{\text {mus }} / A^{*}{ }_{\text {mus }}$ and intraluminal pressure $(\mathbf{A})$, and average radius to circular muscle and total muscle thickness (B), averaged over 8 swallows. The average resting muscle area, $A^{*}$ mus, is $0.40 \mathrm{~cm}^{2}$. The bars indicate SD. The horizontal dotted lines indicate the resting state values $\mathrm{A}^{*}$ mus and $\mathrm{P}^{*}(\mathbf{A})$, and $\mathrm{R}^{*}$ in and $\tau^{*}{ }_{\text {mus }}(\mathbf{B})$. The vertical dotted lines are chosen to match features in the two panels.

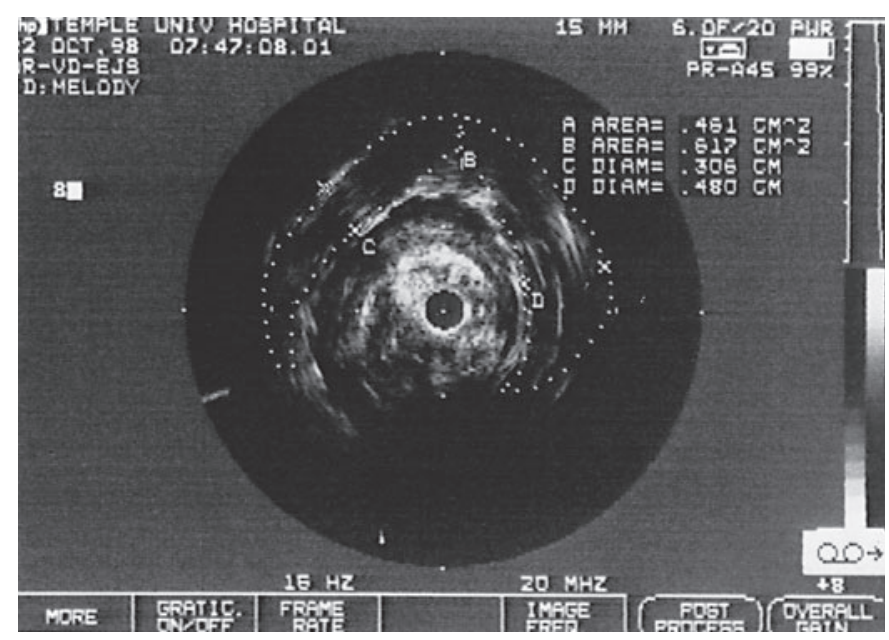

Fig. 12. The area of the LES and the crural diaphragm, which is outlined.

The above study was extended to include the gastric sling fiber/clasp fiber complex by evaluating the anatomic and physiologic relationships between these three components of the gastroesophageal junction high-pressure zone; the LES, the crural diaphragm and the gastric sling fiber/clasp fiber complex, using simultaneous ultrasound and manometry with pharmacologic manipulation [12]. High-frequency ultrasound was co-localized with manometric pressure during breath-holding in inspiration and expiration in normal volunteers. A machine pull-through, of the simultaneous ultrasound and manometry catheter assembly from the stomach into the thoracic esophagus, was performed at a constant velocity. Pull-throughs were repeated before and after intravenous administration of atropine to ablate the pressure from intrinsic sphincter components (LES and gastric sling fiber/clasp fiber complex). The inferior margin of the right crural diaphragm and the initiation of the pull-through (pull-through start position) were used as axial spatial references. The difference between subtractions of the pre-atropine minus the post-atropine pressure profiles (the intrinsic sphincter components) displayed two distinct peaks in both inspiration and expiration, roughly $1.5 \mathrm{~cm}$ apart. The distal peak represented the gastric sling fiber/clasp fiber pressure profile while the proximal peak represented the intrinsic LES pressure profile (fig. 13). By superimposing the three pressure curves (pre-atropine, post-atropine and the pre- minus the post-atropine subtraction curve) it was possible to separate and study each of the components of 


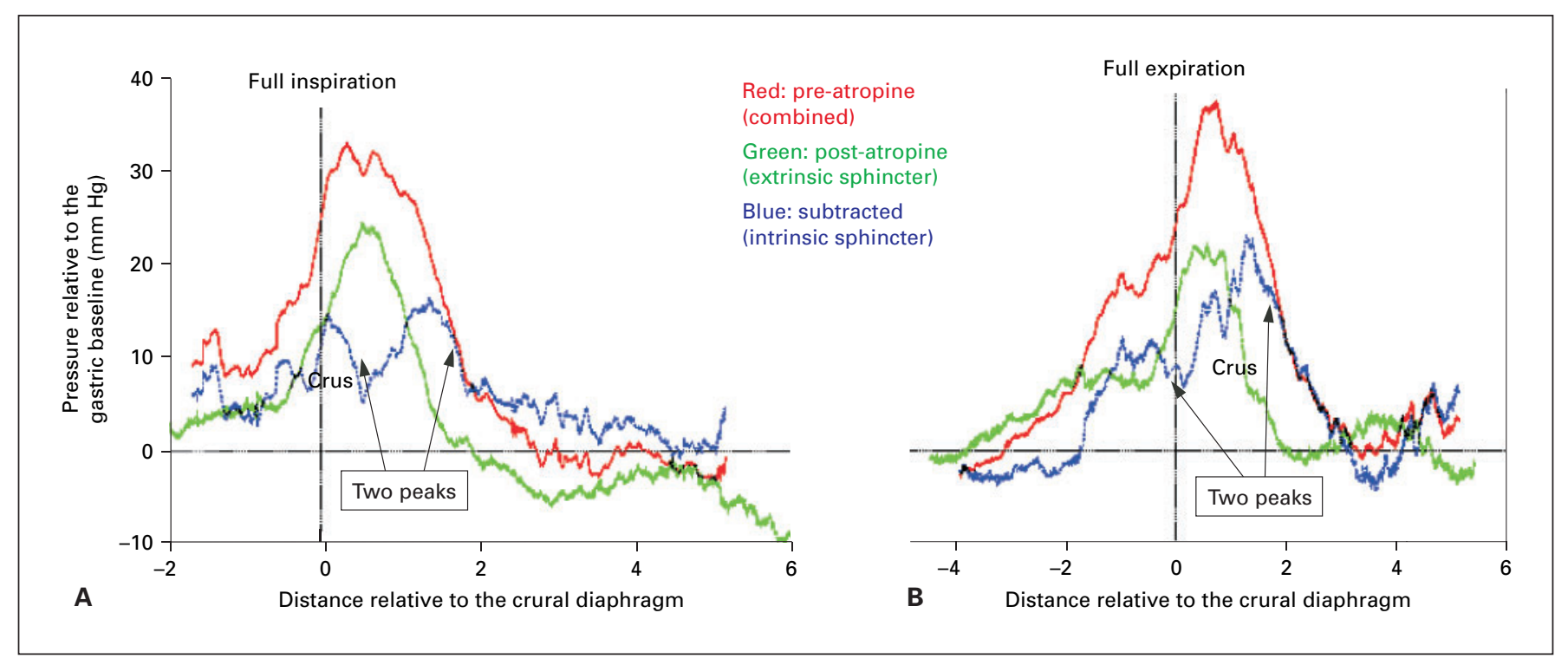

Fig. 13. The combined high-pressure zone and its extrinsic and intrinsic components for both the full inspiration (A) and full expiration respiratory (B) states in 15 normal control subjects. The red line represents the pre-atropine pressure curve (combined external and internal sphincters). The green line represents the post-atropine pressure curve (the extrinsic sphincter). The blue line represents the pre-atropine minus the post-atropine subtraction curve (the intrinsic sphincter components). Note that in both the subtraction curves (full inspiration and full expiration) there are two peaks. The distal peak represents the gastric sling fiber/clasp fiber complex and the proximal curve represents the LES.

the gastroesophageal junction high-pressure zone individually and as an overlapping group for the first time.

Using this technique it was possible to evaluate how each component moved with respect to each of the other components during respiration. It was found that the crus muscle moved approximally $2 \mathrm{~cm}$ between inspiration and expiration, resulting in a $200 \%$ increase in the length of the high-pressure zone with expiration. The inferior margin of the right crus muscle was found to be at the inferior margin of the post-atropine high-pressure zone in both full inspiration and full expiration and extended superiorly $1.83 \mathrm{~cm}$ during full inspiration and $1.73 \mathrm{~cm}$ during full expiration to cover the gastroesophageal junction high-pressure zone, thus indicating that the axial length of the crural diaphragm remained constant during respiration.

In order to confirm and extend the results of the above study, another study was performed in which the crural diaphragm was pharmacologically paralyzed (the external sphincter rather than the internal sphincter as in the study above) in order to define the components of the intrinsic gastroesophageal junction high-pressure zone [13]. Normal control subjects undergoing general anesthesia for non-esophageal surgery were evaluated by placing a simul- taneous ultrasound/manometry probe into the stomach. Cisatracurium was given to paralyze the crural diaphragm. The probe was withdrawn at a constant velocity and ultrasound images and pressure tracings were recorded simultaneously during the inspiratory and expiratory pause on a ventilator. Ensemble averaging of the pressure curves was plotted against the geographic location of the beginning of the crural diaphragm as in the study above. Tracings were compared to normal subjects who were evaluated with and without atropine ablation of the intrinsic components of the gastroesophageal junction high-pressure zone. The ensemble averaging of the patients undergoing crural diaphragm paralysis demonstrated residual pressure profiles from only the intrinsic sphincter components. The same two peaks, in the same relative geographic locations with respect to the crural diaphragm, were seen during crural diaphragm paralysis in the inspiratory pause on the ventilator (fig. 14C, D) as were seen on the atropine subtraction curves in the prior study (fig. 14A, B) [12]. During the expiratory pause on the ventilator, the two peaks were closer together because of the residual volume of air in the lungs and decreased excursion of the crural diaphragm. Once again, the distal intrinsic highpressure zone component was shown to represent the gas- 


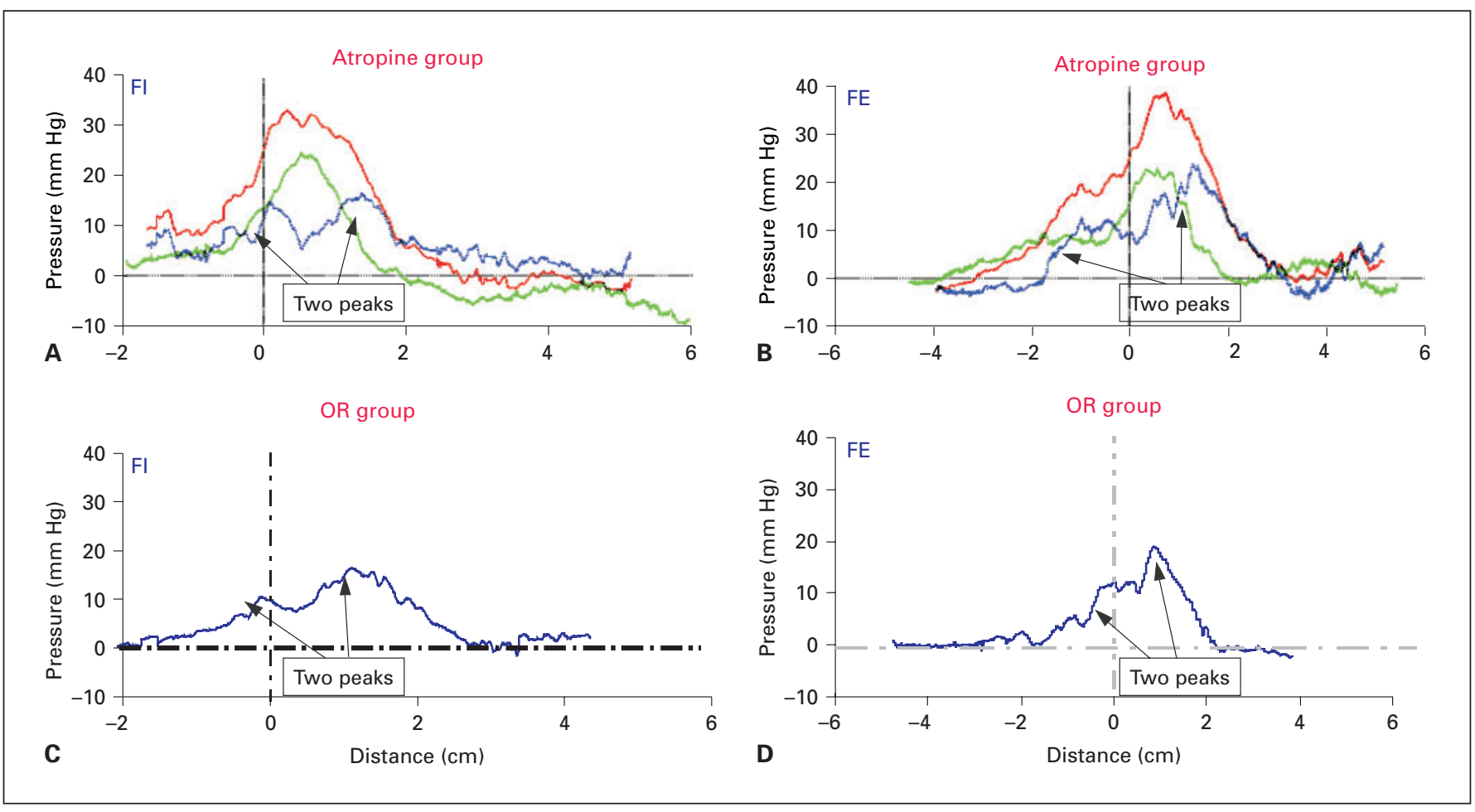

Fig. 14. A, B The ensemble averaging of the normal control group who were evaluated pre- and post-atropine (these are the same curves seen in figure 3). C, D The ensemble averaging of 7 patients undergoing crural diaphragm paralysis (OR group). These graphs represent the residual pressure profiles only from the intrinsic sphincter components. Comparing these curves with the normal control atropine group pressure curves in blue, the same two peaks in both full inspiration (C) and full expiration (D) is demonstrated at the same relative geographic location with respect to the right crural diaphragm. Therefore, the results from crural diaphragm

paralysis are consistent with localization of the intrinsic esophageal sphincter components using smooth muscle ablation with atropine in normal control subjects. The distal peaks represent the pressure profile from the gastric sling fibers while the proximal peaks represent the pressure profile from the intrinsic LES. The peaks are closer together in the OR group than in the atropine group because less air was used to inflate the lungs in the inspiratory pause on the ventilator than in full inspiration and less air was removed from the lungs in the expiratory pause on the ventilator then in full expiration.

tric sling fiber/clasp fiber complex while the proximal component was shown to represent the intrinsic LES.

A third confirmatory study evaluated the anatomic and physiologic relationships between the components of the gastroesophageal junction high-pressure zone using pharmacologic augmentation of the intrinsic high-pressure zone components [14]. The intrinsic and extrinsic sphincters of the gastroesophageal junction high-pressure zone were evaluated using simultaneous ultrasound and manometry with pharmacologic augmentation of the intrinsic (smooth muscle) high-pressure zone components in healthy volunteers. Pull-throughs were repeated before and after oral administration of $100 \mathrm{mg}$ of bethanechol to increase the pressure from the intrinsic (smooth muscle) high-pressure zone components. As in the prior studies, the inferior margin of the right leaf of the right crural

diaphragm was used as a spatial reference. The difference between subtraction curves of the post-bethanechol minus the pre-bethanechol pressure profiles (the augmented intrinsic sphincter components) displayed two distinct peaks in both inspiration and expiration. The distal peak once again represented the gastric sling fiber/clasp fiber pressure profile while the proximal peak represented the intrinsic LES pressure profile (fig. 15).

\section{Lower Esophageal Sphincter}

Lui et al. [1] were the first to demonstrate that the circular and longitudinal smooth muscle of the LES were thicker than the respective muscle layers of the body of the esophagus.

McCray et al. [5] noted that the crural diaphragm was anatomically superimposed on the LES causing compres- 


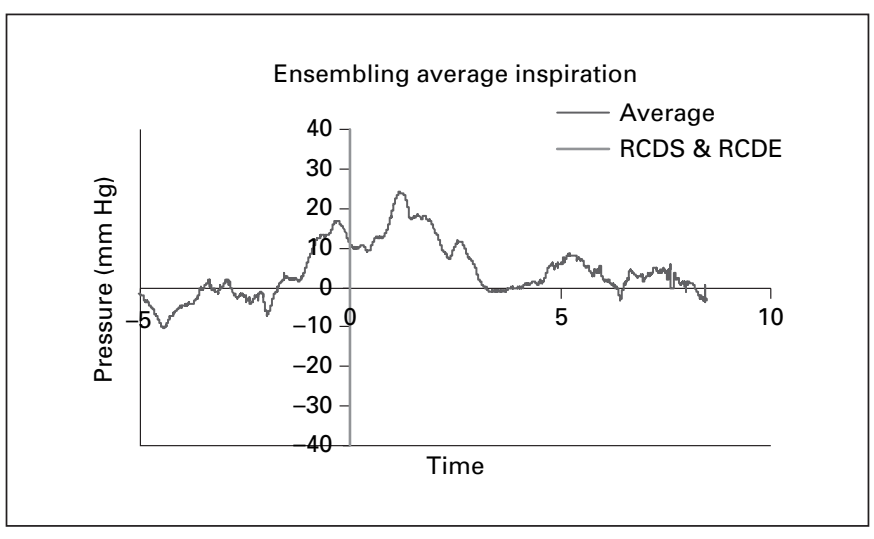

Fig. 15. The post-bethanechol curve minus the pre-bethanechol curve of 14 normal control subjects in full inspiration. Note the two pressure peaks, the distal pressure peak represents the gastric sling fiber/clasp fiber complex while the proximal peak represents the LES. These peaks are in the same relative position as the atropine subtraction curves and the cisatracurium curves above.

sion of the LES so that the anatomy of the LES appears asymmetric, thus accounting for the asymmetry in the pressure profile generated within the LES. The observation that the LES pressure asymmetry disappears in the setting of a hiatal hernia supports this contention.

Ulerich et al. [15] studied the anatomy and physiology of the intrinsic LES esophagogastric segment muscle layers in normal control subjects in greater detail. High-frequency ultrasound was co-localized with manometric pressure with breath-holding under full inspiration and full expiration during a machine pull-through of a simultaneous ultrasound/manometry catheter assembly from the stomach into the thoracic esophagus. For each pullthrough, axial locations of the margins of the right crus muscle were localized and quantified. Pressure was referenced to intragastric pressure. Spatial references used in this study were the inferior margin of the right crus of the diaphragm and the initiation of the pull-through (pullthrough start position). The results showed that the mean muscle thickness in the region of the gastroesophageal junction high-pressure zone increases distally towards the stomach. At each axial location the maximum/minimum thickness ratio was at least $150 \%$. It was also found, using the law of mass conservation, that the esophagogastric segment lengthened during full expiration. It was concluded that the variation in muscle thickness is largest where the diaphragmatic crus contacted the outer wall of the esophagus (fig. 16).

Use of Endoluminal Ultrasound to

Evaluate Gastrointestinal Motility

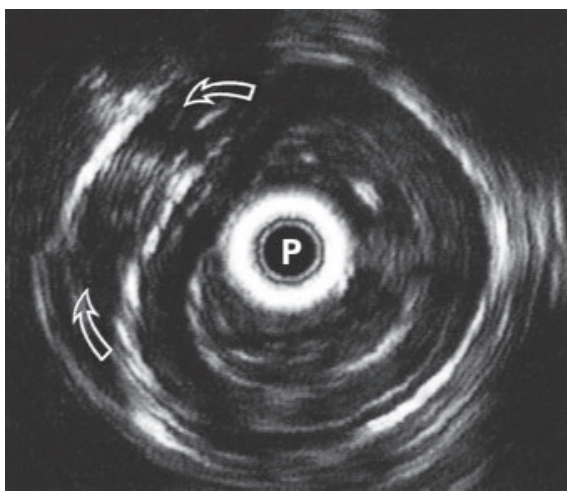

Fig. 16. An image of the LES and crural diaphragm (arrows). Note the crural diaphragm is located next to the LES and causes compression of the LES leading to asymmetry.

\section{Upper Esophageal Sphincter}

In contrast to the rest of the sphincter muscles in the gastrointestinal tract, the upper esophageal sphincter is less well studied. The unique characteristics of the upper esophageal sphincter have made precise evaluation of its anatomy and physiology difficult. Events at the striated muscle upper esophageal sphincter during swallowing occur quickly relative to events in the rest of the upper gastrointestinal tract.

Miller et al. [16] used simultaneous high-resolution endoluminal sonography and manometry to evaluate upper esophageal sphincter motion, anatomy, morphology, and pressures at baseline resting levels and during swallowing, and quantitate the amount of pressure generated at the upper esophageal sphincter due to the mechanical conformation of the upper esophageal sphincter and active resting myogenic tone.

The upper esophageal sphincter and its surrounding structures were evaluated in normal subjects and human cadavers. The upper esophageal sphincter musculature (cricopharyngeus) was identified as a hypoechoic C-shaped structure surrounding the mucosa and submucosa in the high-pressure zone of the pharyngoesophageal region, forming an angle of $107 \pm 19^{\circ}$ in normal subjects. Two posterior cricoarytenoid muscles (hypoechoic oval structures adjacent to the posterior aspect of the cricoid cartilage, and separated from the upper esophageal sphincter and cricoid cartilage by hyperechoic connective tissue), the hyperechoic adventitia, fatty tissue surrounding the upper esophageal sphincter musculature, and the hypoechoic spinal musculature were identified as well (fig. 17). 
Fig. 17. The ultrasound image of the upper esophageal sphincter is shown on the left. Note the Cshaped configuration of the cricopharyngeal muscle $(\mathrm{CP})$. The posterior cricoaryetnoid muscles (PCA) are labeled. The transducer (T) appears in the center of the lumen surrounded by the mucosal/submucosal complex. The cricoid cartilage (CC) appears as a hyperechoic structure, which causes acoustic shadowing. On the right is the simultaneous manometric pressure.

Fig. 18. A series of images (A-H) during a pullthrough from the upper esophagus into the upper esophageal sphincter. Note that the transition of the morphology of the mural structure from an oval-shaped esophageal body to a C-shaped upper esophageal sphincter. The final image (I) shows the ultrasound transducer (fixed in position in the esophagus), originally positioned within the upper esophageal sphincter, is now in the upper esophagus (UE) during a swallow. This is due to elevation of the upper esophageal sphincter during swallowing.
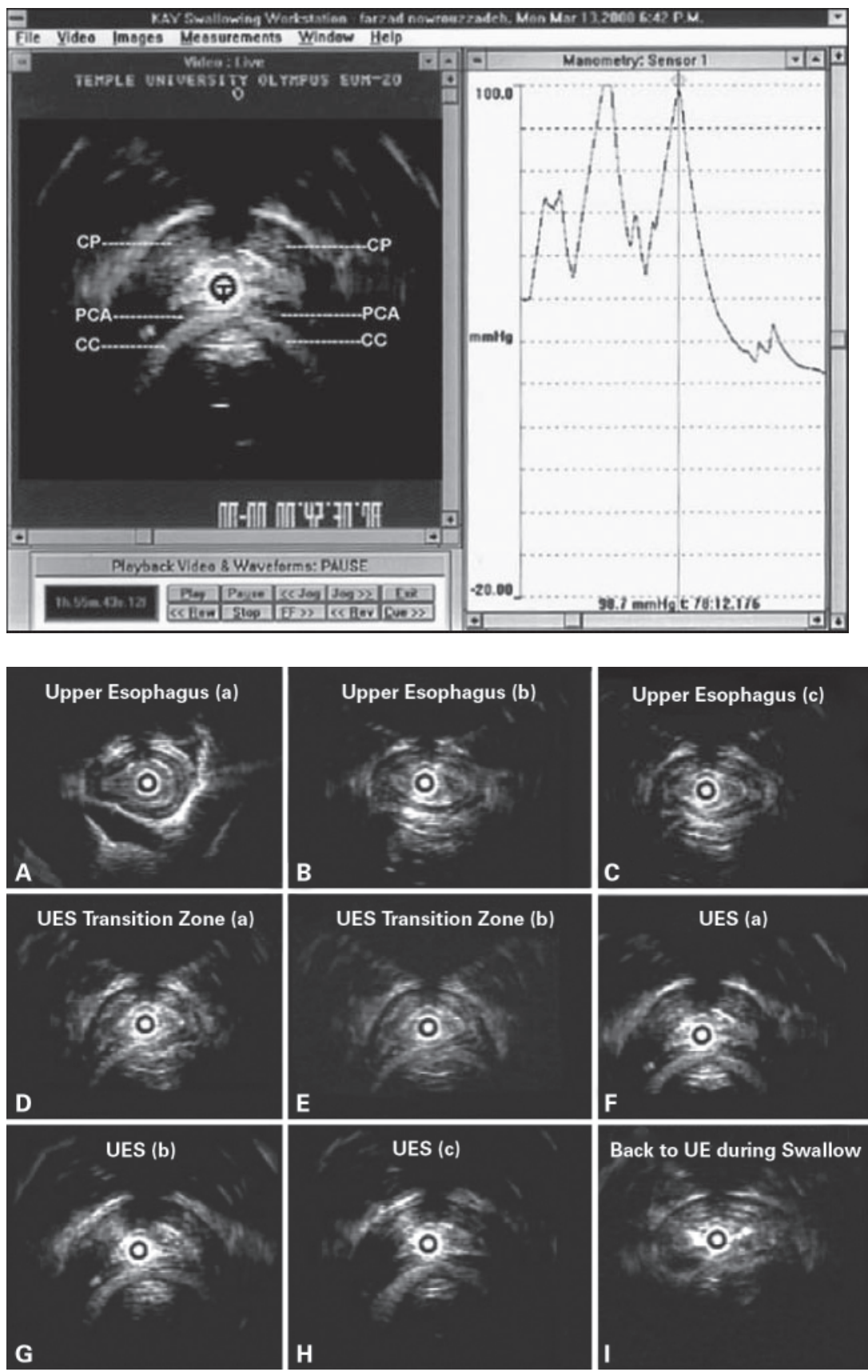

The mean peak resting upper esophageal sphincter pressure was $74 \mathrm{~mm} \mathrm{Hg}$, with a total cross-sectional area of $0.87 \pm 0.33 \mathrm{~cm}^{2}$, both of which were significantly greater than the muscle cross-sectional area and pressure within the upper esophageal body. During swallowing, the upper esophageal sphincter moved in an orad direction pulling the upper esophagus along with it and then dropped back to its original position (fig. 18). Localizing the upper esophageal sphincter sonographically in the ca- davers, the peak upper esophageal sphincter pressure was found to be $19.7 \pm 10.0 \mathrm{~mm} \mathrm{Hg}$. This demonstrated that about a quarter $(26.5 \%)$ of the peak pressure in the upper esophageal sphincter was due directly to the morphologic, anatomic, and structural configuration of the upper esophageal sphincter, with the rest of the pressure being due to active resting muscle tone within the upper esophageal sphincter. 
Fig. 19. A High-resolution endoluminal ultrasound sonogram from the area of the LES in a patient with achalasia. Note the marked increase in thickness of the circular smooth muscle (CM) and longitudinal smooth muscle compared with the image in figure 5. B An image of the dilated body of the esophagus in a patient with achalasia (EL = esophageal lumen, $\mathrm{T}=$ transducer $)$.
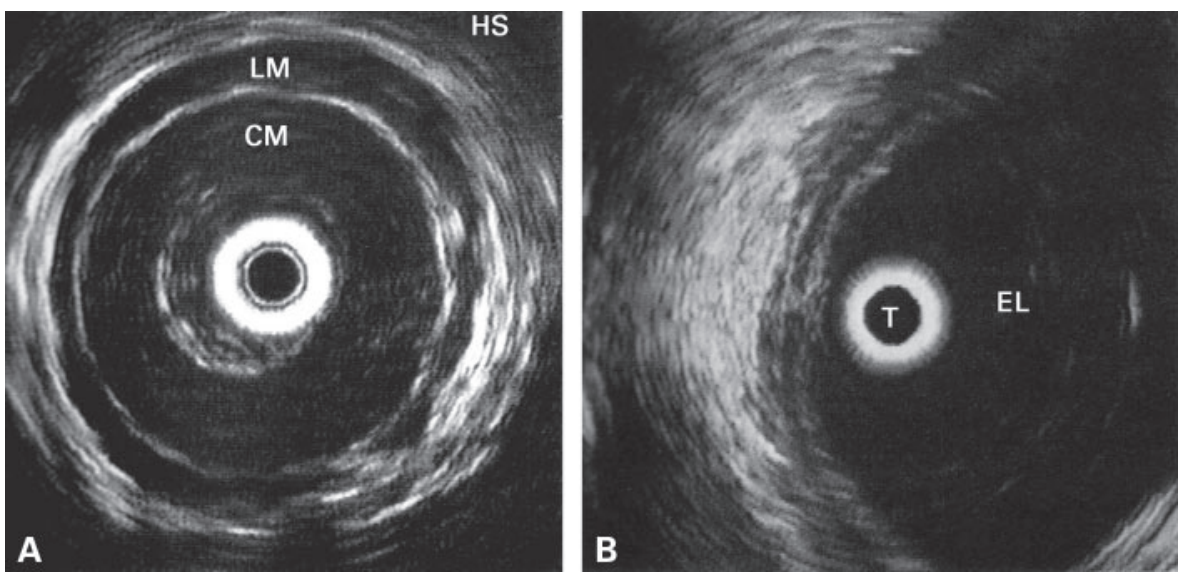

\section{Esophageal Pathology}

Once the normal sonographic anatomy of the esophagus was defined in vivo, using the high-resolution (20$\mathrm{MHz}$ ) endoscopic ultrasound transducer, it became possible to look for abnormalities in patients with various esophageal disorders.

\section{Achalasia}

Miller et al. [17] demonstrated that in patients with achalasia, there was a mean increase in the thickness of the circular smooth muscle, longitudinal smooth muscle, and total muscle in the region of the LES (fig. 19). However, it was also concluded that a thickened muscularis propria found on high-resolution $(20-\mathrm{MHz})$ endoscopic ultrasound could not be used to differentiate clinically between patients with achalasia and normal controls due to marked variability in muscle thickness in achalasia and an overlap in muscle width between patients with achalasia and normal individuals. Upon retrospective review of clinical, manometric, and radiographic data, patients with achalasia with marked thickening of the muscularis propria could not be differentiated from achalasia patients without muscular thickening.

Schiano et al. [18] utilized high-resolution endoluminal ultrasonography to assess esophageal wall damage after pneumatic dilation and botulinum toxin injection in the treatment of achalasia. Patients with achalasia were treated by either botulinum toxin injection at the LES or pneumatic dilation. The layers of the esophageal wall were imaged by high-resolution $(20-\mathrm{MHz})$ endoscopic ultrasound transducer before, immediately after, and $24 \mathrm{~h}$ after either the pneumatic dilation or botulinum toxin injection. High-resolution $(20-\mathrm{MHz})$ endoscopic ultrasound revealed a similar pretreatment mucosa-submu- cosa diameter in both treatment groups. There was a significant increase in the mucosa-submucosa diameter thickness of the esophageal wall immediately after pneumatic dilation as compared with the pretreatment measurement. This increase in thickness was consistent with edema of the esophageal wall. This twofold increase normalized within $24 \mathrm{~h}$ after the procedure was performed, indicating a transient traumatic insult. In contrast, there was no significant difference in the mucosa-submucosa after botulinum toxin injection.

\section{Scleroderma}

The esophagus is the most frequently affected gastrointestinal organ in scleroderma, and is often associated with significant clinical symptomatology. Miller et al. [19] conducted a three-part study utilizing high-resolution endoluminal ultrasonography in patients with scleroderma to evaluate esophageal involvement. In the first part, esophageal autopsy specimens were compared both histologically and sonographically from patients with scleroderma and without scleroderma. In the second part of the study, the esophagi of normal control subjects and scleroderma patients were evaluated in vivo using high-resolution (20-MHz) endoluminal ultrasound to determine whether or not there were ultrasound abnormalities consistent with the findings on the autopsy specimens. In the third part of the study, the degree of ultrasound abnormalities seen in the scleroderma patients was quantified and compared to the grade of manometric abnormalities in the peristaltic contraction.

The first part of the study revealed that patients with scleroderma have a hyperechoic abnormality in the normally hypoechoic muscularis propria. This finding was not present in autopsy specimens from non-scleroderma 
Fig. 20. A, $\mathbf{A}^{\prime}$ Post-mortem ultrasound of the esophagus in patients with SSc. Hyperechoic regions extend into the circular smooth muscle layer (CM). At some locations, the circular smooth muscle layer is completely obliterated by hyperechoic echoes. Longitudinal smooth muscle (LM) is clearly seen. $\mathrm{T}=$ Transducer. $\mathbf{B}, \mathbf{B}^{\prime}$ Histological sections stained with Masson's trichrome stain, from the post-mortem esophagus of the same area seen in $\mathbf{A}, \mathbf{A}^{\prime}$. The $\mathrm{CM}$ is infiltrated by fibroconnective tissue (blue). Longitudinal smooth muscle LM shows minimal fibrosis.
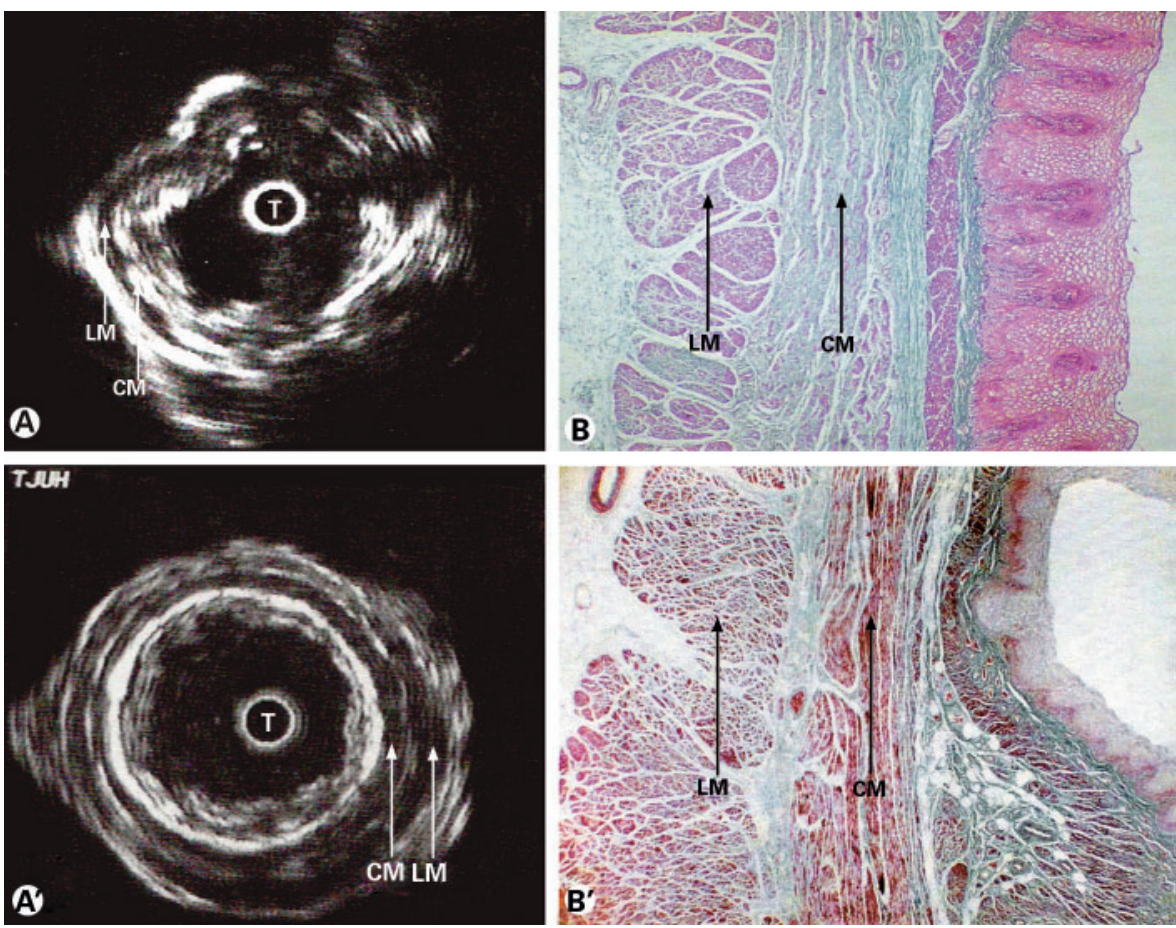

patients (fig. 20). This hyperechoic abnormality correlated with the presence of fibrosis on histologic sections from autopsy specimens. The second part of the study confirmed the presence of this hyperechoic abnormality in the scleroderma patients in vivo as opposed to the normal controls. A grading system was developed to stratify the degree of fibrosis (hyperechogenicity) found in the patients with scleroderma and to correlate the grade of fibrosis with the manometric abnormities. A significant correlation $(r=0.89)$ was found between the grade of manometric abnormality and the degree of hyperechoic abnormality (representing degrees of fibrosis) (fig. 21). The findings of the study call into question the pathogenesis and etiology of the esophageal abnormalities in scleroderma demonstrating that fibrosis of the muscularis propria may be the primary event in this disorder.

\section{Gastroesophageal Reflux Disease (GERD)}

Miller et al. [20], using simultaneous ultrasound and manometry, discovered that an abnormality of the gastric sling fiber/clasp fiber complex exists in patients with GERD. The distal pressure profile due to the gastric sling fiber/clasp fiber complex, seen in normal control subjects, was lacking in patients with GERD (fig. 22). It was also found that the lack of this pressure profile increased the compliance of the gastroesophageal junction high-pressure zone, allowing pathologic distension of this area.
The relative contributions of each of the components of the gastroesophageal junction high-pressure zone were evaluated and compared in GERD patients and normal control subjects, in order to determine which abnormalities predispose patients to reflux. High-frequency endoluminal ultrasound and simultaneous manometry was used to evaluate patients with documented GERD during inspiration and expiration as in the previous studies in normal volunteers. As described above, the difference between pre- and post-atropine pressure profiles in normal volunteers (the intrinsic sphincter components) displayed two peaks in inspiration and expiration. However, in GERD patients the subtraction curve between the preand post-atropine pressure profiles demonstrated that the distal peak pressure profile (gastric sling fiber/clasp fiber complex) was totally absent in both the inspiratory and expiratory phases of all of the GERD patients (fig. 22). The proximal peak representing the LES was present in the same axial location relative to the crural diaphragm as in the normal volunteers. It was concluded that GERD patients lack the pressure profile consistent with the gastric sling fiber/clasp fiber complex.

Simultaneous ultrasound and manometry has also been used to evaluate the etiology of symptoms in GERD. Sustained esophageal contraction of the longitudinal muscle of the esophagus was seen prior to the onset of heartburn. Acid infusion into the esophagus, which 

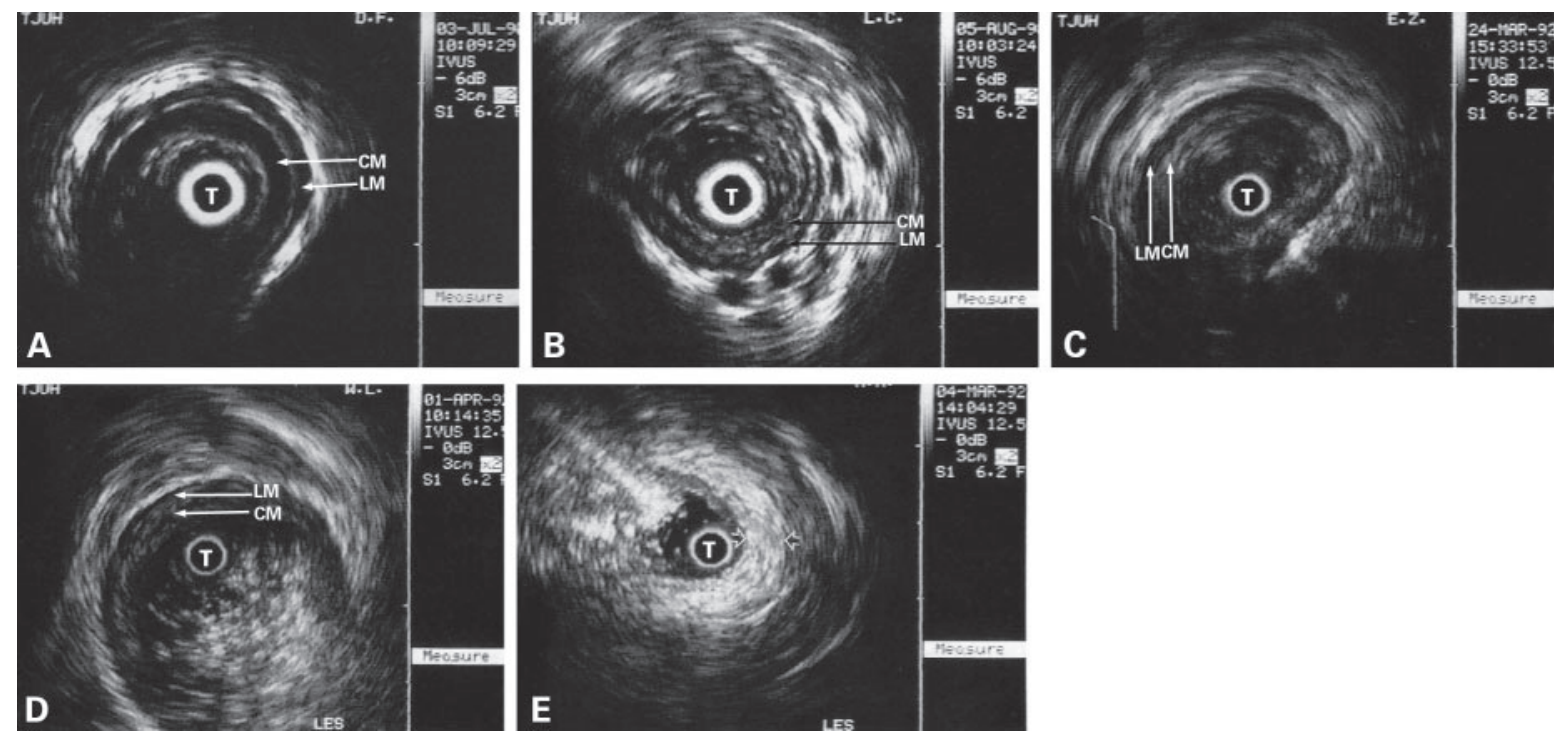

Fig. 21. A Grade 1: all layers appear normal. There is no hyperechoic abnormality in either layer of the muscularis propria. B Grade 2: hyperechoic abnormality in the muscularis propria involving but not obliterating the inner circular smooth muscle (CM). Longitudinal smooth muscle (LM) appears normal. C Grade 3: hyperechoic abnormality in the muscularis propria obliterating the CM but not involving the outer LM. D Grade 4: hyperechoic abnormality in the muscularis propria obliterating the CM and involving the LM but not obliterating the longitudinal smooth muscle. E Grade 5: complete replacement of the entire muscularis propria with hyperechoic abnormality in the muscularis propria (arrows). $\mathrm{T}=$ Transducer.

caused a heartburn sensation, was also associated with contraction of the longitudinal muscle of the esophagus. Reflux-induced distention of the esophagus during liquid reflux episodes can be assessed using endoluminal ultrasound and ultrasound imaging can determine the nature of the reflux episode, i.e. liquid, gas or both [21].

Lui et al. [1] demonstrated the consequences of severe GERD on ultrasound by showing loss of the mucosa and a marked thickening of the submucosa in patients with ulcerative esophagitis on endoluminal ultrasound (fig. 23).

\section{Endoscopic Treatment of GERD}

Surgical and endoscopic treatments for GERD focus on altering the structure of the gastroesophageal segment by directly modifying its mechanical properties. These methods rely on the augmentation of the natural barriers to reflux.

To further define the effects of endoscopic plication in patients with GERD, Miller et al. [22] performed a study to define the mechanism of action of endoscopic plications. High-frequency ultrasound and simultaneous manometry was used to evaluate GERD patients in a fashion similar to the previous studies. Eight of ten patients (80\%) had a significant clinical benefit after endoscopic plica- tion with elimination or with a marked decrease in symptoms. Pre-plication, all of the patients demonstrated the absent gastric sling fiber/clasp fiber pressure profile described above. Post-plication the distal pressure profile was re-established in the GERD patients at the same location as the distal pressure peak in normal volunteers (fig. 24).

Dai et al. [23] were able to localize endoscopic plications with respect to the crural diaphragm and LES in order to determine the physiologic effects of the plication. Simultaneous ultrasound and manometry was performed in patients with GERD before and after endoscopic plication. The catheter assembly was placed in the stomach and withdrawn through the gastroesophageal junction high-pressure zone at a constant velocity during breathholding. Ultrasound images were analyzed for the configuration of the plications, depth of sutures, location of the plications with respect to the crural diaphragm and changes in the pressure profile. Three-dimensional reconstruction of the ultrasound images was performed. It was found that the plications appeared as hypoechoic round structures on 2-dimensional ultrasound and hypoechoic spherical structures on 3-dimensional ultrasound (fig. 25). The sutures within the plications appeared as 
Fig. 22. A An ensemble averaged graph from 7 patients with GERD. The red curve represents the pre-atropine pressure. The green curve represents the post-atropine pressure due to the crural diaphragm. The blue curve represents the subtraction curve of the red curve minus the green curve, or the intrinsic components of the gastroesophageal junction high-pressure zone. Note: the blue curve contains only one peak, representing the LES. The distal peak from the gastric sling fiber/clasp fiber complex is completely absent. B The distal peak is seen in normal control subjects. This represents the gastric sling fiber/clasp fiber complex.

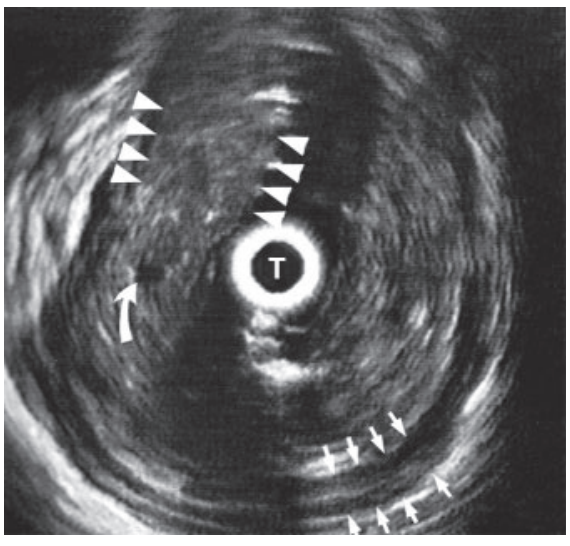

Fig. 23. In a patient with acute esophagitis, there is marked increase of the third, hyperechoic layer in the distal esophagus. The curved arrow represents a small-dilated blood vessel within this thickened region (arrowheads). Arrows represent the normal muscularis propria. $\mathrm{T}=$ Transducer.

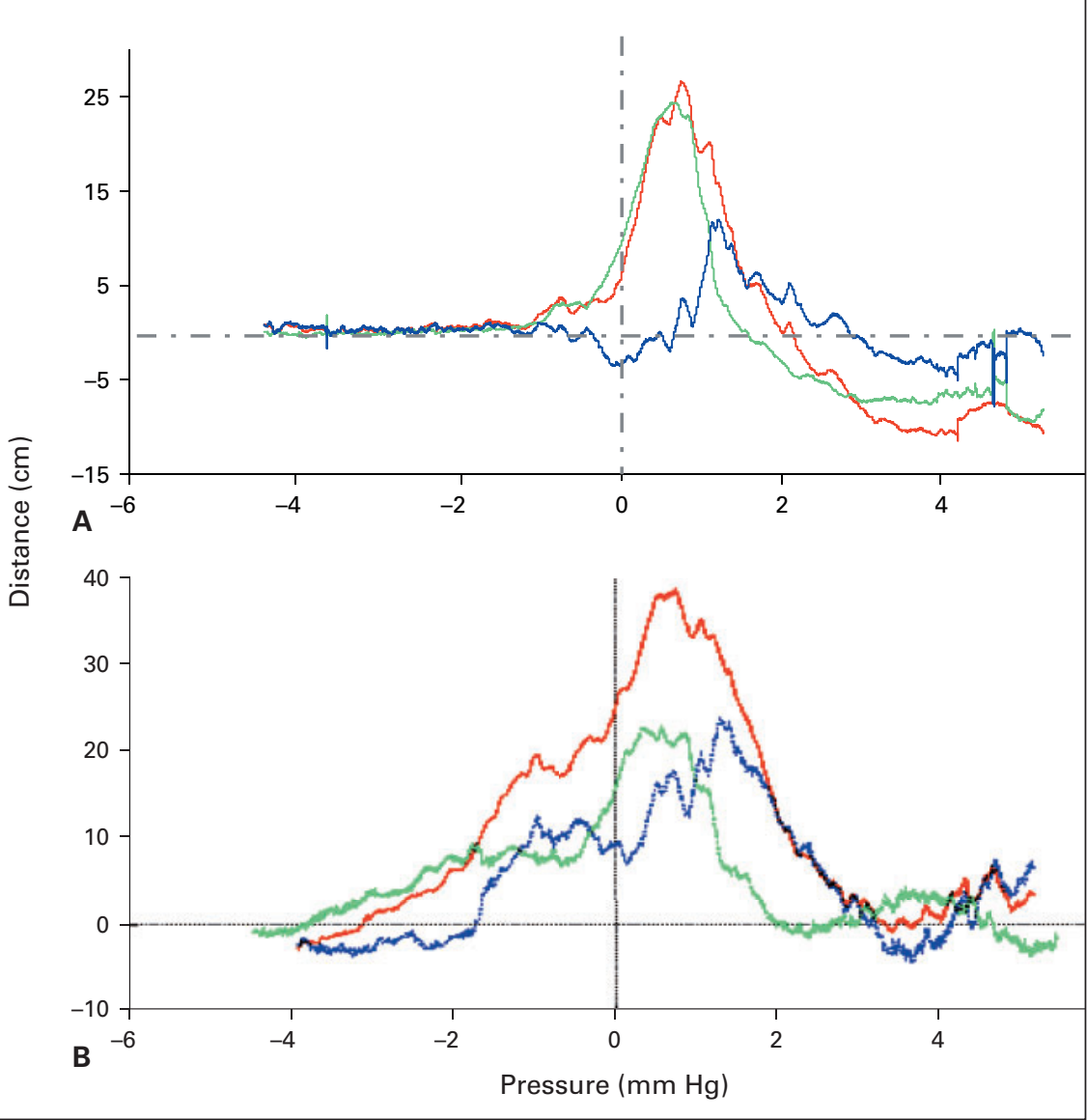

hyperechoic lines. The majority of sutures were localized to the submucosa. The majority of the plications were located at or just below the right crus of the diaphragm. The distal portion of the pressure profile in the gastroesophageal junction high-pressure zone was lengthened in the area of the plications (fig. 26).

\section{Surgical Treatment of GERD}

The sonographic and manometric characteristics of Nissen fundoplication were evaluated in order to determine the effects of gastric smooth muscle tone vs. physical mechanical compression on the antireflux barrier after Nissen fundoplication. Dai et al. [24] used a simultaneous high-frequency ultrasound transducer and manometry catheter, which was placed into the stomach in patients after Nissen fundoplication. The catheter was pulled through the area of the Nissen fundoplication into the esophagus at a constant velocity, before and after the administration of intravenous atropine during both inspiration and expiration (breath-holding). An investigator 
Fig. 24. The ensemble average of the pressure profile from the 8 GERD patients who responded symptomatically to endoscopic plication. The distal pressure peak (gastric sling fiber/clasp fiber complex) was re-established during full inspiration in the GERD patient's post-EndoCinch at the same location as the distal pressure peak in the normal volunteers. Note: the red curve represents the post-EndoCinch pressure profile and the green curve represents the pre-EndoCinch pressure profile, while the blue curve is the subtraction curve of postEndoCinch minus pre-EndoCinch pressure profile. The vertical dashed line represents the start of the crural diaphragm, used as an axial reference mark.

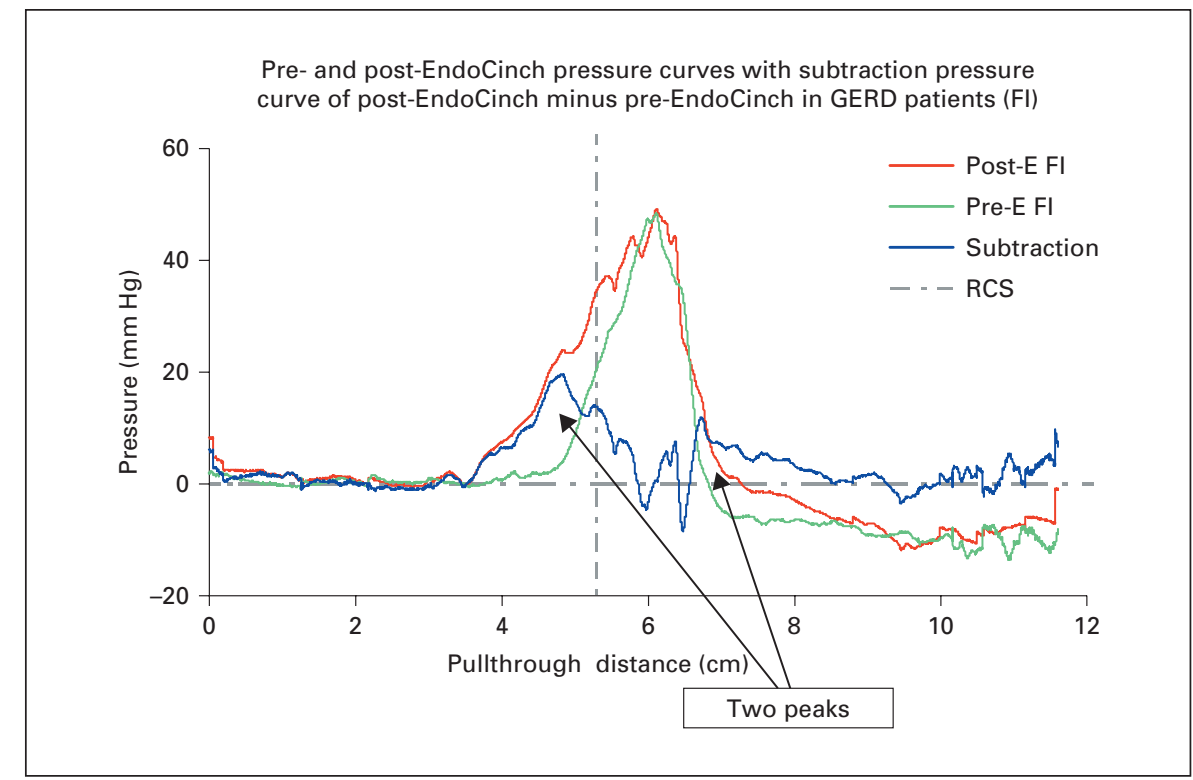

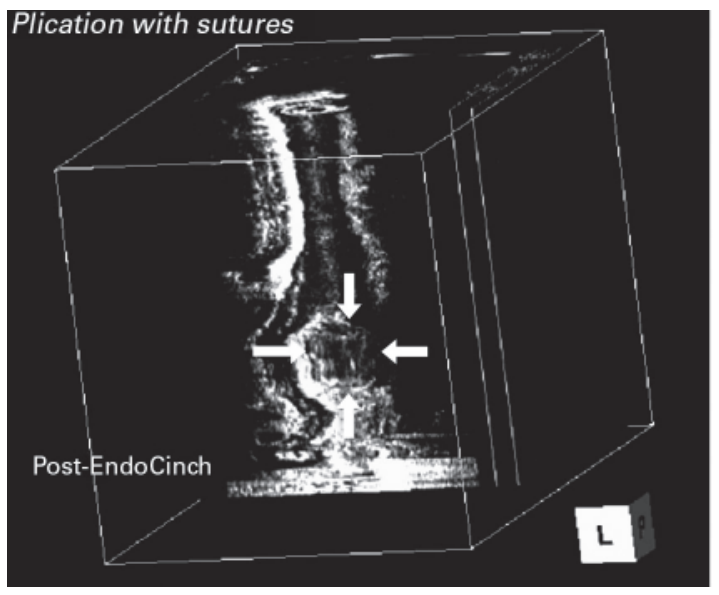

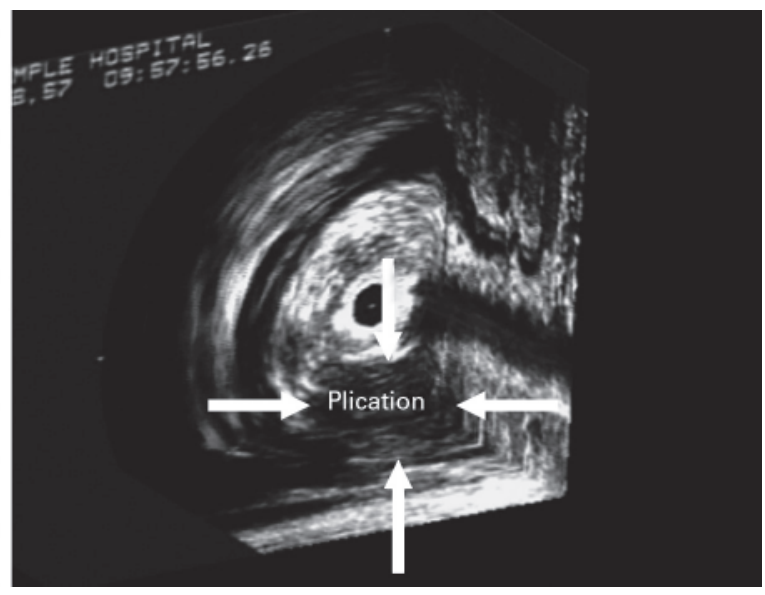

Fig. 25. Three-dimensional reconstructions of the area of the gastric plications (arrows). The plication appears as a hypoechoic spherical structure with hyperechoic sutures within the plication.

blinded to the pressure profile marked the beginning of the Nissen fundoplication and the beginning of the crural diaphragm on ultrasound. The beginning of the Nissen fundoplication was defined as the first area at which multiple layers of the gastric wall could be imaged (fig. 27). The beginning of the crural diaphragm was defined by imaging the muscle of the crural diaphragm outside the wall of the stomach or esophagus (fig. 10). The area under the pressure curve was evaluated between the beginning of the Nissen fundoplication and the beginning of the crural diaphragm and the peak pressure within the Nissen fundoplication was assessed. The results showed that the area under the pressure curve decreased by $44 \%$ ( $p<$ 0.0001 ) and the peak pressure decreased by $32 \%$ (from $80.4 \pm 37.2$ to $54.8 \pm 24.3), \mathrm{p}<0.0001$, within the Nissen fundoplication after the administration of atropine (fig. 28). It was concluded that the antireflux barrier due to a Nissen fundoplication is in large part due to the tonic contraction of the gastric smooth muscle in the wrap of the Nissen fundoplication and not simply due to the mechanical effect of the wrap compressing the esophagus. Based on these data it was hypothesized that the Nissen 


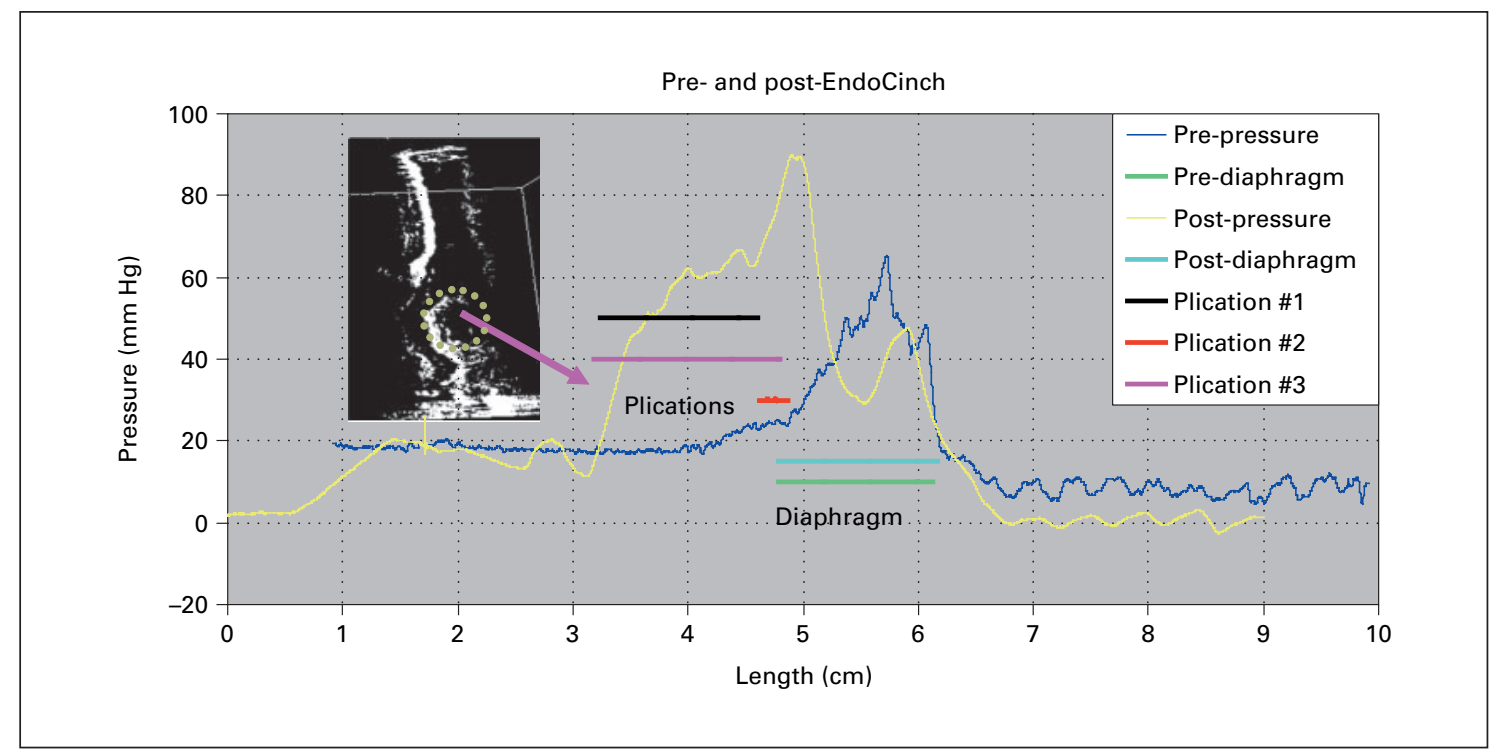

Fig. 26. The pressure profiles through the gastroesophageal junction high-pressure zone pre- and post-EndoCinch in a patient with GERD referenced to the right crural diaphragm. Note: the blue curve represents the preEndoCinch pressure profile, while the yellow curve represents the post-EndoCinch pressure profile. The horizontal lines represent the locations of the endoscopic plications (red, pink and green lines), and the right crural diaphragm (light blue, and light green lines) located by ultrasound imaging.
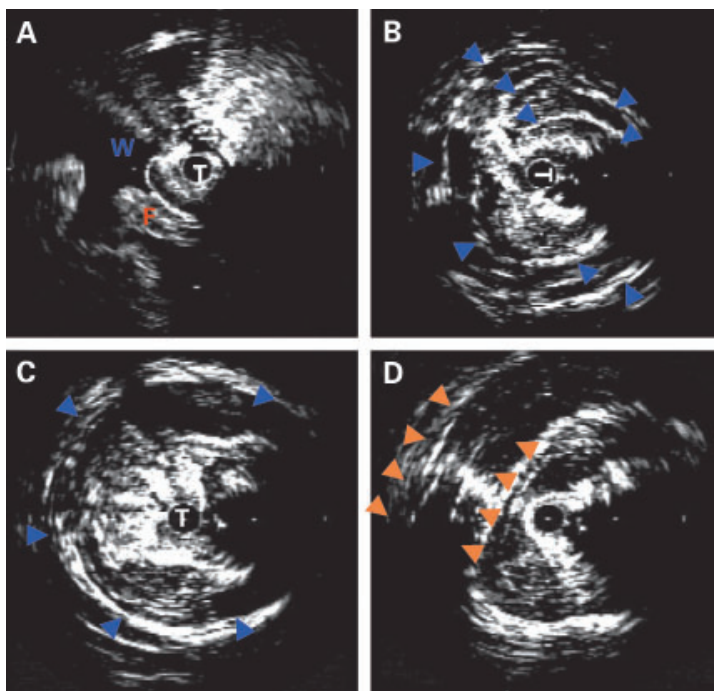

Fig. 27. A Cross-sectional sonogram through the funds of the stomach. The mucosa folds (F) of the stomach and the echo-free water $(\mathrm{W})$ are shown in the lumen. $\mathrm{T}=$ Transducer. B A cross-sectional sonogram through the Nissen fundoplication. Multiple layers (arrows) of the stomach wall are shown as alternately hypoand hyperechoic structures around the ultrasound transducer (T). C A cross-sectional sonogram through Nissen fundoplication. D A cross-sectional sonogram of the gastric esophageal junction showing the crural diaphragm (arrows) as a belt-like hypoechoic structure. $\mathrm{T}=$ Transducer. fundoplication, like endoscopic plication, prevents reflux by artificially bolstering the area of the defective gastric sling fiber/clasp fiber complex and that tonic contraction of the muscle in the gastric wrap is an important factor in generating this antireflux barrier.

\section{Barrett's Esophagus}

Barrett's esophagus is a consequence of chronic GERD and is therefore included in this discussion of motility disorders. Adrian et al. [25] demonstrated that sonographically, a thickened second hypoechoic layer of the mucosa is seen in Barrett's esophagus, independent of the presence of dysplasia (fig. 29). However, there is no conclusive evidence that endoscopic ultrasound is effective at diagnosing or grading dysplasia in Barrett's esophagus.

The principal role of endoscopic ultrasound in evaluating patients with Barrett's-associated dysplasia is to identify patients who may be candidates for endoscopic ablative (endoscopic mucosal resection, photodynamic therapy) [26-28] therapies. Hence, the endoscopist may be able to distinguish between localized tumors and tumors with invasion beyond the mucosa, or metastases to lymph nodes. Periesophageal, celiac, and posterior mediastinal lymphadenopathy can also be sampled with fineneedle aspiration. 
Fig. 28. This graph shows an overlap of the pre- and post-atropine pressure curves. The pre-atropine pressure curve is in blue. The post-atropine pressure curve is in red. Note: the area under the curve between lines $\mathrm{N}$, $\mathrm{D}$ and $\mathrm{B}$ is significant less for the post-atropine pressure curve (red) than for the preatropine pressure curve (blue). $\mathrm{N}=$ Beginning of the Nissen, $\mathrm{D}=$ beginning of the diaphragm, $\mathrm{B}=$ baseline pressure.

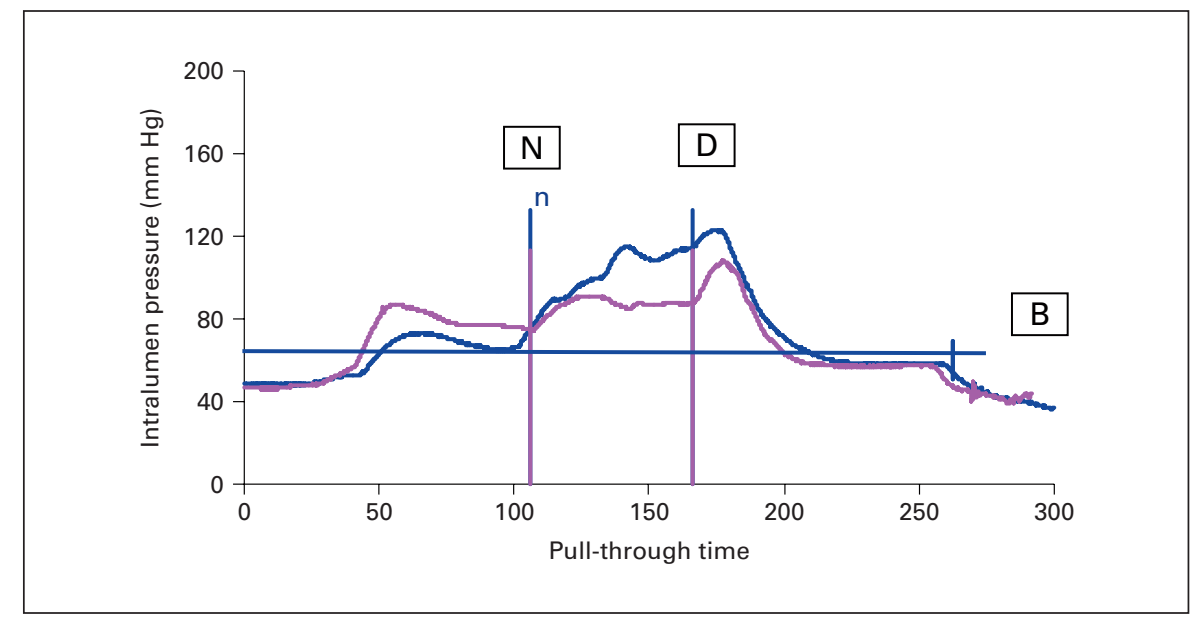

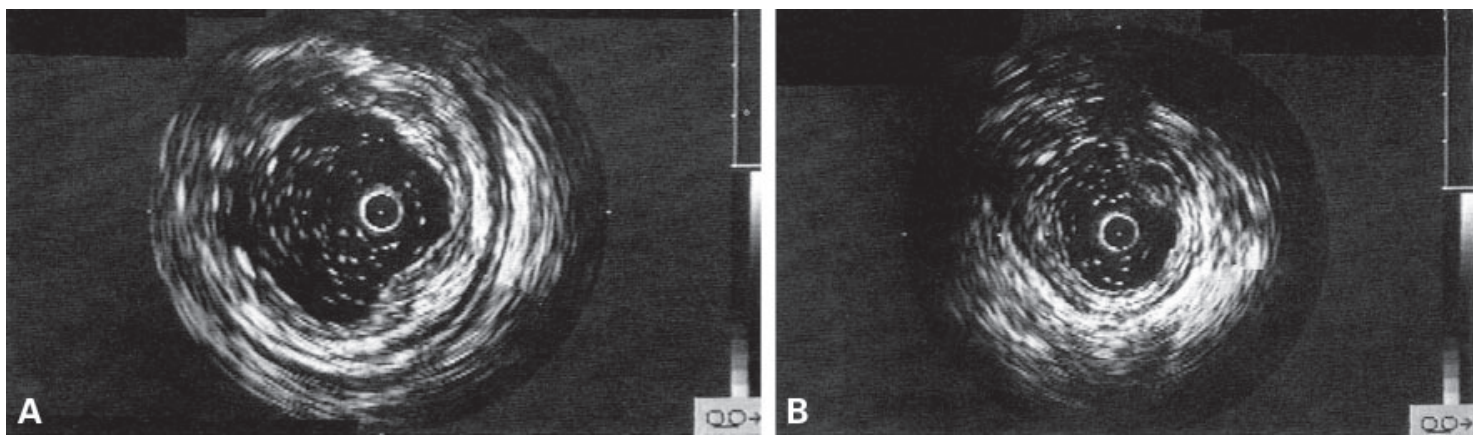

Fig. 29. High-resolution endoluminal sonography images from the esophagus of a patient with Barrett's metaplasia. A In the area of metaplasia the second hypoechoic layer can be seen to be thicker than the first hyperechoic layer. B In normal squamous mucosa above Barrett's metaplasia in the same patient the second hypoechoic layer is thinner than the first hyperechoic layer.

\section{Esophageal Motility Disorders}

Longitudinal smooth muscle may be involved in various disorders of the esophagus. The esophagus is one of the sources of 'angina-like pain'. Balaban et al. [29] has shown that simultaneous recording of esophageal pressure and ultrasound images revealed a sustained contraction of the longitudinal muscle of the esophagus prior to the onset of chest pain events. However, there was no evidence of a sustained increase in the esophageal pressure during these contractions. Although this temporal correlation does not prove causality between the two events, pharmacologically induced chest pain (with edrophonium hydrochloride/tensilon) was also associated with a sustained longitudinal muscle contraction.

The new technique of 3-dimensional time-resolved reconstruction developed by Dai et al. [8] has the potential to simplify the evaluation of sustained esophageal con- tractions as demonstrated in diffuse esophageal spasm and non-cardiac chest pain.

\section{The Biomechanics of the Gastroesophageal Junction High-Pressure Zone}

Stresses and strains can be calculated from data generated using simultaneous ultrasound and manometry during bolus distension of the esophagus (fig. 30) [30]. To further evaluate the pathophysiologic mechanism of the absent gastric sling fiber/clasp fiber pressure profile in GERD patients, the geometry, stiffness and wall tension of the gastroesophageal junction in the resting state and during luminal opening among normal subjects, GERD patients, and the same GERD patients after endoscopic plication was compared (fig. 31). Deglutitive inhibition 
Fig. 30. Pressure difference, radius, total muscle layer thickness, total muscle layer area, log ratio $\left(1 / \ln \left[1+\tau / R^{2}\right]\right)$ and stress against time for a normal control subject during swallowing.

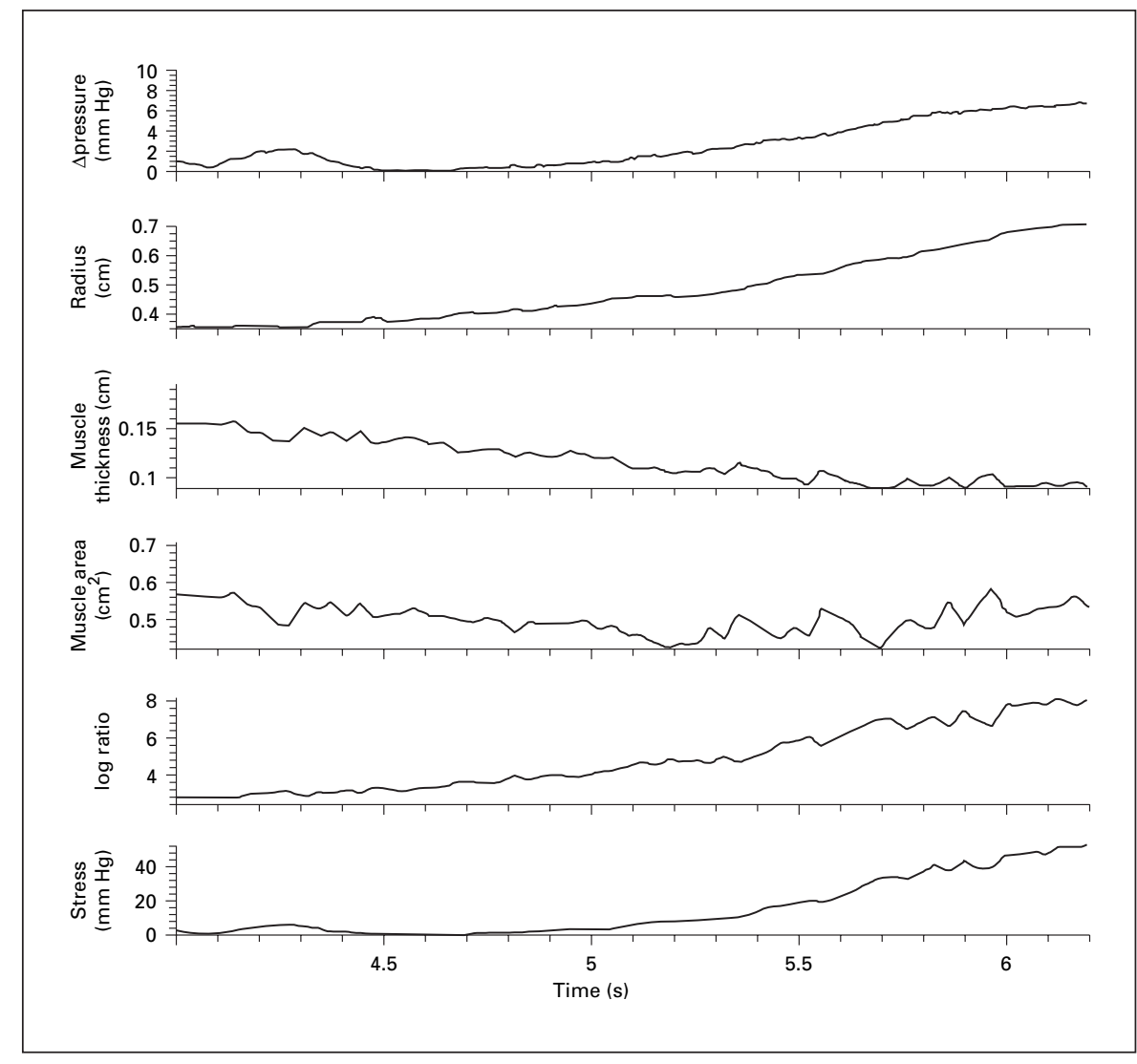

during swallowing was used to measure the mechanical properties of the normal, abnormal, and endoscopically plicated gastroesophageal junction. Using high-frequency endoluminal ultrasound concurrently with manometry, cross-sectional lumen and wall geometries were quantified with intraluminal pressure during bolus distension of the gastroesophageal junction with water in normal controls and in patients' pre- and post-endoscopic plication approximately $1 \mathrm{~cm}$ above the plications. Esophageal lumen radii, areas, and muscle thicknesses were measured during opening. A force balance equation was applied to calculate average wall tension. The results demonstrated that the stiffness of the muscle wall surrounding the gastroesophageal junction was approximately sixfold less in the GERD patients than in the normal control subjects. After placement of the endoscopic plications, the average stiffness was found to approximate normal values. It was concluded that the plications increased the stiffness of the esophagogastric segment, but only at the distal-most end of the gastroesophageal junction high-pressure zone (the gastric cardia) due to the placement of the plications between the cardia and the esophagocardiac junction. This is the exact site of the missing gastric sling fiber/clasp fiber pressure profile, thus demonstrating that the missing gastric sling fiber/clasp fiber pressure profile changes the compliance and stress-to-strain relationships of the distal gastroesophageal junction high-pressure zone.

\section{Anal Sphincter Complex}

Both anal manometry and endoluminal ultrasonography have been used to study the anal sphincter complex: manometry to measure the pressure of the anal sphincters, and endoluminal ultrasound to determine muscle anatomy and thickness. The anal canal, the terminal 3$4 \mathrm{~cm}$ of the gastrointestinal tract, is surrounded by two primary muscles, the internal anal sphincter and the external anal sphincter. The internal anal sphincter is the innermost muscle of the complex and is a continuation of circular smooth muscle from the muscularis propria of the rectum. The outermost external anal sphincter is composed of bands of striated muscle arising from a downward extension of the puborectalis muscle. These structures have been sonographically imaged utilizing 5.0- to $7.5-\mathrm{MHz}$ endorectal probes (with diameter of approxi- 
mately $2 \mathrm{~cm}$ ) positioned within the anal canal. The anatomic information obtained has provided a basis for assessment of anal canal abnormalities such as sphincter disruptions, fistulas, and anal cancer.

Alexander et al. [31] used high-resolution endoluminal sonography of the anal sphincter complex using a 9-Fr catheter with a $12.5-\mathrm{MHz}$ transducer revealed a hypoechoic mucosa, hyperechoic submucosa, and hypoechoic internal anal sphincter (as well as a part of the external anal sphincter). The images obtained were measured at 8 octants for the internal anal sphincter and first band of external anal sphincter. It was clearly shown that the internal anal sphincter itself varies in thickness and cross-sectional area, depending on the location within the anal canal. In the proximal portion of the internal anal sphincter, the cross-sectional surface area and thickness of the muscle are at a minimum. The cross-sectional surface area and thickness of the internal anal sphincter increase to a maximum at approximately the center of the internal anal sphincter and then decrease again gradually. The internal anal sphincter ends at the distal aspect of the anal canal prior to the termination of the external anal sphincter (fig. 32).

Prior studies had endeavored to determine the correlation between resting anal pressure and sphincter thickness. The data had been inconclusive, with some studies finding a positive correlation and others finding no correlation. Wong et al. [6] used simultaneous anal manometry and endoluminal ultrasonography within the anal canal to demonstrate that resting anal pressure and crosssectional areas of the anal sphincters vary throughout the anal canal, first increasing and then decreasing. By ultrasound, the internal anal sphincter cross-sectional area is $>10 \mathrm{~mm}$ proximal to the peak resting anal pressure and corresponds closely with the maximum external anal sphincter cross-sectional area. There was a strong correla- tion demonstrated between resting anal pressure and the sum of the internal and external sphincter cross-sectional area. Thus, in contrast to previous reports, this study suggested that the external anal sphincter might have a greater contribution to the resting anal pressure than previously thought. Scar tissue and fibroconnective tissue within the internal anal sphincter appears as a hyperecho-

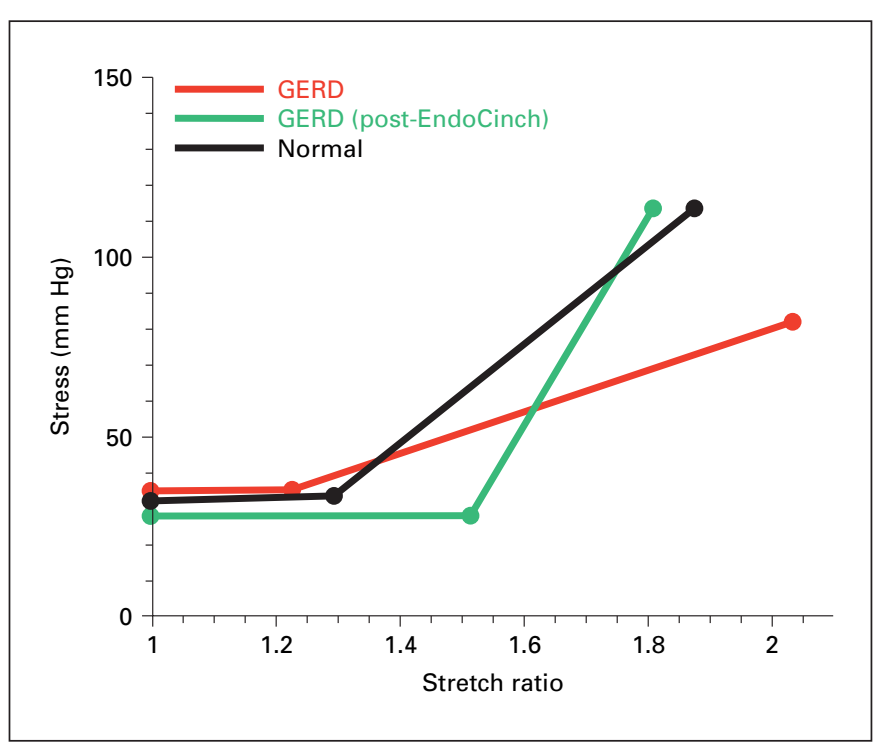

Fig. 31. The stress-to-stretch ratio plots for normal control subjects (black), patients with GERD pre-EndoCinch (red) and the same GERD patients' post-EndoCinch (green). Note: the stress-to-stretch ratio is shifted to the right in the GERD patients compared to the normal controls, but shifts back to the left after the endoscopic plications. The results demonstrate that the stiffness of the muscle wall surrounding the gastroesophageal junction was approximately six times lower in the GERD patients than in the normal control subjects but that after placement of endoscopic plications, the average stiffness was found to approximate normal values.
Fig. 32. A A transanal ultrasound image with the use of a catheter-based $20-\mathrm{MHz}$ transducer (hypoechoic mucosa (M), hyperechoic submucosa (S), internal anal sphincter (IAS), and a portion of external anal sphincter (EAS). B A patient with a history of anal canal surgery, transanal ultrasound image using a catheter-based 20-MHz probe delineates a hyperechoic defect within the internal anal sphincter (IAS). T = Transducer.

Use of Endoluminal Ultrasound to Evaluate Gastrointestinal Motility
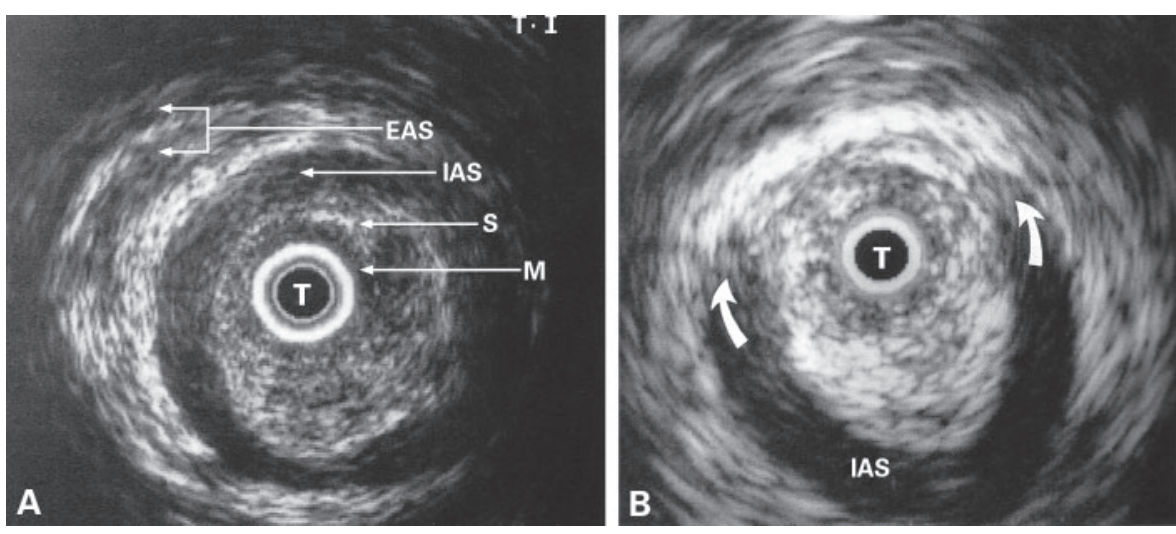

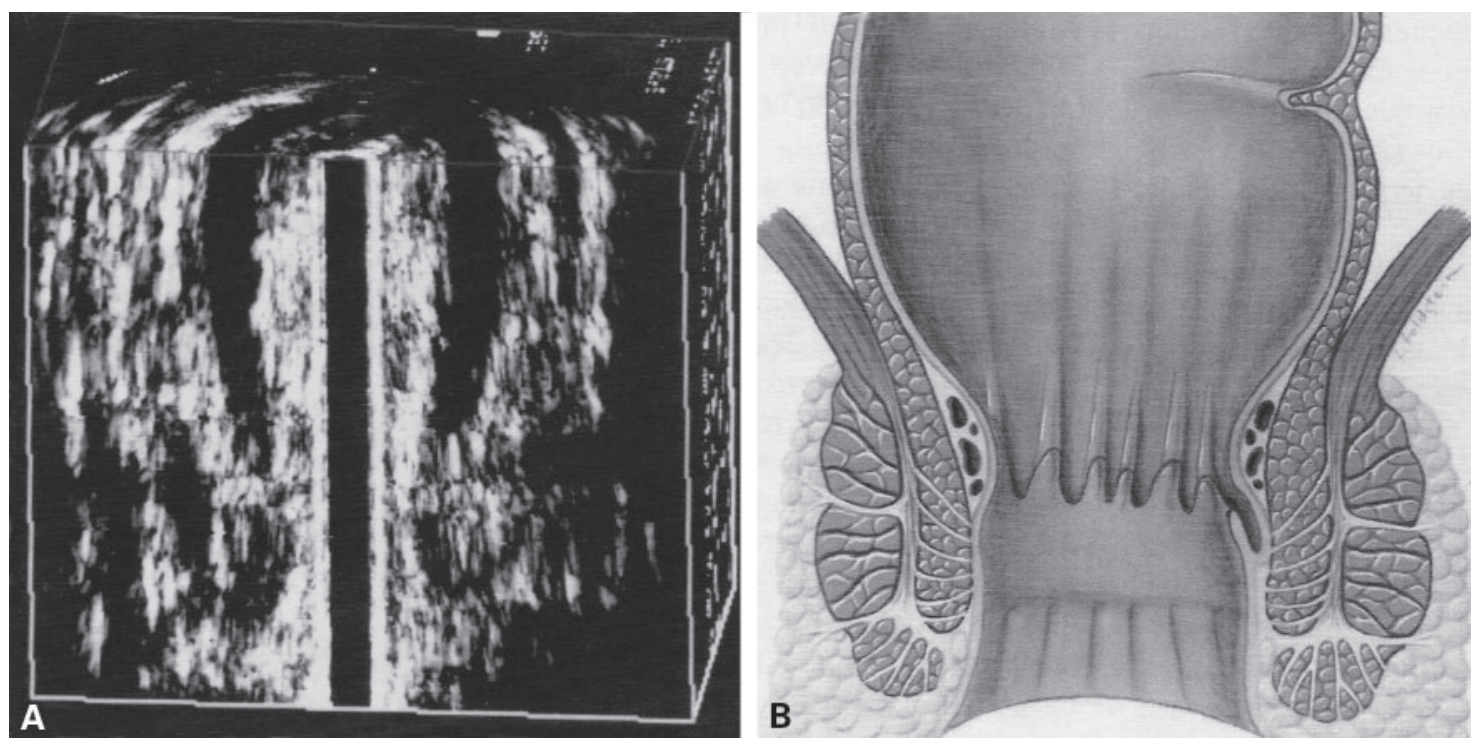

Fig. 33. A Three-dimensional transanal ultrasound image of the anal sphincter complex using a catheter-based 20-MHz ultrasound transducer. B Diagrammatic representation of the anal sphincter complex. It can be seen that the muscle layers iin the diagram correlate with the muscle layers in the three-dimensional ultrasound image.

ic defect within the normally hypoechoic internal anal sphincter (fig. 32). These abnormalities can be located very precisely using 3-dimensional imaging, and the length and severity of the defect can be accurately evaluated. Three-dimensional imaging technology can provide surgeons with a precise map of the longitudinal and transverse location of the sphincter defect prior to surgically reconstructing the internal anal sphincter (fig. 33). In addition, these sphincter defects correlate very well with manometric abnormalities within the sphincter.

\section{Conclusion}

The use of high-frequency ultrasound transducers in the gastrointestinal tract has already yielded remarkable findings concerning the anatomy, physiology and pathophysiology of the gastrointestinal tract and of various motility disorders. These transducers have made completely invisible portions of the gastrointestinal tract (the longitudinal smooth muscle, muscles of the upper esophageal sphincter, components of the gastroesophageal junction high-pressure zone, and the muscle of the anal sphincter complex) accessible to investigation. Use of simultaneous ultrasound and manometry has allowed the exploration of the normal physiology of peristaltic contraction. The components of the high-pressure zone of the distal and proximal esophagus have been isolated and the movement of these components has been studied individually and as a group. Various esophageal motility disorders have been investigated including achalasia, scleroderma, Barrett's esophagus and diffuse esophageal spasm. The possible etiology of the symptoms of esophageal chest pain and heartburn (sustained esophageal contractions of the longitudinal smooth muscle), have been studied. The possible underlying pathophysiology of GERD (the missing gastric clasp and sling fiber pressure profile) has been explored. Three-dimensional high-frequency ultrasound imaging has allowed the peristaltic contraction sequence to be viewed in a completely new and unique manner. The biomechanics of both esophageal contraction and the gastroesophageal junction high-pressure zone have been investigated and the mechanical advantage of esophageal shorting has been studied. The mechanism of action of standard surgical and newer endoscopic therapies for GERD has been defined. Future applications of this technology are limited only by our imagination. 


\section{References}

1 Liu JB, Miller LS, Goldberg BB, et al: Transnasal US of esophagus: preliminary morphologic and function studies. Radiology 1992; 184:721-727.

2 Miller LS, Liu JB, Klenn PJ, et al: High-frequency endoluminal ultrasonography of the esophagus in human autopsy specimens. J Ultrasound Med 1993;12:563-566.

-3 Liu J, Miller LS, Goldberg BB: Endoluminal ultrasound in gastroenterology: Application of new technology. Clin Diagn Ultrasound 1994; 29:185-215.

4 Miller LS, Liu JB, Colizzo FP, et al: Correlation of high frequency esophageal ultrasonography and manometry in the study of esophageal motility. Gastroenterology 1995; 105: 832-837.

-5 McCray WH Jr, Chung C, Parkman HP, Miller LS: Use of simultaneous high-resolution endoluminal sonography and manometry to characterize high-pressure zone of distal esophagus. Dig Dis Sci 2000;45:1660-1666.

-6 Wong RF, Bonapace ES Jr, Chung CY, Liu JB, Parkman HP, Miller LS: Simultaneous endoluminal sonography and manometry to assess anal sphincter complex in normal subjects. Dig Dis Sci 1998;43:2363-2372.

7 Liu JB, Miller LS, Bagley DB, Bonn J, Forsberg F, Goldberg BB: Three-Dimensional Endoluminal Ultrasound. London, Dunitz, 1998, pp 325-346.

-8 Dai Q, Liu JB, Brasseur JG, Thangada VK, Thomas B, Parkman H, Miller LS: Volume (three-dimensional) space-time reconstruction of esophageal peristaltic contraction by using simultaneous US and manometry. Gastrointestinal Endosc 2003;58:913-919.

-9 Miller LS, Liu JB, Colizzo FP, et al: Correlation of high-frequency esophageal ultrasonography and manometry in the study of esophageal motility. Gastroenterology 1995;105: 832-837.

10 Nicosia MA, Brasseur JG, Liu JB, Miller LS: Local longitudinal muscle shortening of the human esophagus from high-frequency ultrasonography. Am J Physiol 2000;281:G1022G1033.

-11 Pal A, Brasseur JG: The mechanical advantage of local longitudinal shortening on peristaltic transport. J Biomech Eng 2002;124:94-100.

12 Ulerich R, Miller L, Dai Q, Brasseur JG: Pharmacologic separation of the gastro-esophageal segment into extrinsic and intrinsic sphincters. Gastroenterology 2003;124:A259-S1746.
13 Dai QS, Soliman AS, Dilipkumar P, Thangada V, Brasseur J, Chung C, Ulerich R, Parkman H, Thomas B, Miller LS: Simultaneous ultrasound and manometry during pharmacologic paralysis of the crural diaphragm allows spatial localization of the two components of the intrinsic gastroesophageal junction high-pressure zone. Gastroenterology 2004;126:A636W1437.

14 Neela D, Korimilli A, Dai Q, Brasseur J, Parkman H, Miller L: Pharmacologic augmentation of the intrinsic sphincter components, isolates the gastric sling fibers, crural diaphragm and intrinsic lower esophageal sphincter at the gastroesophageal junction (abstract). A 520, T 1641, Gastro 2005.

15 Ulerich R, Dai Q, Miller L, Brasseur J: Detailed three-dimensional anatomy of the human gastroesophageal segment. Gastroenterology 2003;124:A259-S1747.

16 Miller LS, Dai Q, Sweitzer BA, Thangada V, Kim JK, Thomas B, Parkman H, Soliman AM: Evaluation of the upper esophageal sphincter using simultaneous high-resolution endoluminal sonography and manometry. Dig Dis Sci 2004;49:703-709.

17 Miller LS, Liu JB, Barbarevich, C, Baranowski, RJ, Dhuria M, Schiano TD, Goldberg B, Fisher RS: High-resolution endoluminal sonography in achalasia. Gastrointest Endosc 1995;42: 454-459.

18 Schiano TD, Fisher RD, Parkman HP, Cohen S, Dabezier M, Miller LS: Use of high-resolution endoscopic ultrasonography to assess esophageal wall damage after pneumatic dilation and botulinum toxin injection to treat achalasia. Gastrointest Endosc 1996;44:151157.

19 Miller LS, Liu JB, Klenn PJ, Holahan MP, Varga J, Feld RI, Troshinsky M, Jimenez SA, Castell DO, Goldberg B: Endoluminal ultrasonography of the distal esophagus in systemic sclerosis. Gastroenterology 1993;105:31-39.

20 Miller LS, James B, Ulerich R, Thomas B, Thomas A, Dai Q: A new theory to explain the pathophysiology of GERD. Pharmacological separation of the gastroesophageal junction high-pressure zone demonstrates an absent gastric sling fiber pressure profile in patients with GERD. Gastroenterology 2004;126: A503-T1741.
21 Pehlivanov N, Liu J, Mittal RK: Sustained esophageal contraction: A motor correlate of heartburn symptom. Am J Physiol 2001;281: G743-G751.

22 Miller LS, Dai Q, Dimitriou J, Schiffer B, Brasseur J: Endoscopic plication (EndoCinch) repairs a physiologic defect in patients with gastroesophageal reflux disease: absent pressure profile due to the gastric sling fibers. Gastroenterology 2004;126:A330-M1420.

23 Dai Q, Brasseur JG, Dimitriou J, Chung C, Thomas A, Asfari W, Miller LS: Two- and three-dimensional endoluminal ultrasound localization of endoscopic plications with simultaneous manometry (location of plications and depth of sutures) (abstract). Gastrointest Endosc 2004;59:AB 244.

-24 Dai Q, Chung CY, Nowrouzzadeh F, Parkman H, Brasseur J, Thangada V, Miller LS: Simultaneous ultrasound and manometry in the evaluation of Nissen fundoplication. Gastroenterology 2003;124:A418-M2113

25 Adrain AL, Ter Han-Chuan, Cassidy MJ, Schiano TD, Liu JB, Miller LS: High-resolution endoluminal sonography is a sensitive modality for the identification of Barrett's metaplasia. Gastrointest Endosc 1997;46:147-151.

-26 May A, Gossner L, Gunter E, et al: Local treatment of early cancer in short Barrett's esophagus by means of argon plasma coagulation: initial experience. Endoscopy 1999;31:497-500.

27 Sharma P: Endoscopic mucosal resection of early cancer and high-grade dysplasia in Barrett's esophagus. Gastrointest Endosc 2002;55: 137-139.

28 Wolfsen HC, Woodward TA, Raimondo M: Photodynamic therapy for dysplastic Barrett's esophagus and early esophageal adenocarcinoma. Mayo Clin Proc 2002;77:1176-1181.

29 Balaban D, Yamamoto Y, Liu J, et al: Sustained esophageal contraction: a marker of esophageal chest pain identified by intraluminal ultrasonography. Gastroenterology 1999; 116:29-37.

30 Schiffner BJ, Miller L, Dai Q, Dimitriou J, Brasseur JG: Opening stiffness and geometry of the esophago-gastric segment in health, with GERD, and after endoscopic surgery (abstract). A 396, M1808, Gastro 2005.

- 31 Alexander AA, Miller LS, Liu JB, Feld RI, Goldberg B: High-resolution endoluminal sonography of the anal sphincter complex. J U1trasound Med 1994;13:281-284. 